TRANSACTIONS OF THE

AMERICAN MATHEMATICAL SOCIETY

Volume 352, Number 5, Pages 2087-2119

S 0002-9947(99)02295-3

Article electronically published on May 3, 1999

\title{
THE GEOMETRY OF FIXED POINT VARIETIES ON AFFINE FLAG MANIFOLDS
}

\author{
DANIEL S. SAGE
}

\begin{abstract}
Let $G$ be a semisimple, simply connected, algebraic group over an algebraically closed field $k$ with Lie algebra $\mathfrak{g}$. We study the spaces of parahoric subalgebras of a given type containing a fixed nil-elliptic element of $\mathfrak{g} \otimes k((\pi))$, i.e. fixed point varieties on affine flag manifolds. We define a natural class of $k^{*}$-actions on affine flag manifolds, generalizing actions introduced by Lusztig and Smelt. We formulate a condition on a pair $(N, f)$ consisting of $N \in \mathfrak{g} \otimes k((\pi))$ and a $k^{*}$-action $f$ of the specified type which guarantees that $f$ induces an action on the variety of parahoric subalgebras containing $N$.

For the special linear and symplectic groups, we characterize all regular semisimple and nil-elliptic conjugacy classes containing a representative whose fixed point variety admits such an action. We then use these actions to find simple formulas for the Euler characteristics of those varieties for which the $k^{*}$-fixed points are finite. We also obtain a combinatorial description of the Euler characteristics of the spaces of parabolic subalgebras containing a given element of certain nilpotent conjugacy classes of $\mathfrak{g}$.
\end{abstract}

\section{INTRODUCTION}

Let $G$ be a semisimple, simply connected, algebraic group over an algebraically closed field $k$, and let $\mathfrak{g}$ be its Lie algebra. The set of Borel subalgebras of $\mathfrak{g}$ forms a smooth, projective variety which we denote by $\mathcal{B}$. For any nilpotent element $N$ in $\mathfrak{g}$, the set of Borel subalgebras containing $N$ is a closed subvariety $\mathcal{B}_{N}$ of $\mathcal{B}$. These varieties have been studied extensively and have interesting applications to representation theory. For example, Springer and several others, including Ginzburg, Kazhdan, Lusztig, Slodowy, Joseph, Borho, and MacPherson, have defined representations of the Weyl group $W$ in the homology (or cohomology) of the $\mathcal{B}_{N}$ 's. Moreover, all irreducible representations of $W$ may be obtained from the Springer representations in the top homology of the $\mathcal{B}_{N}$ 's as $N$ runs over the nilpotent conjugacy classes of $\mathfrak{g}$. In fact, when $G=S L_{n}(k)$, these representations are precisely the irreducible representations of $W$.

Kazhdan and Lusztig have introduced the study of the affine analogue of these fixed point varieties on the flag manifold, which they anticipate will have applications to the character theory of semisimple $p$-adic groups. They have also used affine techniques to extend to all nilpotent orbits of $\mathfrak{g}$ a map defined by Carter and Elkington from a certain class of nilpotent orbits into the conjugacy classes of $W$. Let $F=k((\pi))$ be the field of formal power series in one variable with $A=k[[\pi]]$

Received by the editors November 1, 1997.

1991 Mathematics Subject Classification. Primary 14L30, 20G25.

Key words and phrases. Fixed point varieties on affine flag manifolds, Iwahori subalgebras, parahoric subalgebras, lattices. 
its ring of integers. We then extend scalars to form $G(F), G(A), \mathfrak{g}_{F}=\mathfrak{g} \otimes_{k} F$, and $\mathfrak{g}_{A}=\mathfrak{g} \otimes_{k} A$. Fix a Borel subalgebra $\mathfrak{b} \in \mathcal{B}$. An Iwahori subalgebra of $\mathfrak{g}_{F}$ is any $G(F)$-conjugate of the pullback $\hat{\mathfrak{b}}$ of $\mathfrak{b}$ under the natural projection $\mathfrak{g}_{A} \rightarrow \mathfrak{g}$; we denote the space of Iwahori subalgebras by $\hat{\mathcal{B}}$. If $B \subset G$ corresponds to $\mathfrak{b}$ with preimage $\hat{B} \subset G(A)$ under the map $G(A) \rightarrow G$, then $\hat{\mathcal{B}}$ may be identified with the quotient space $G(F) / \hat{B}$. The set $\hat{\mathcal{B}}$ has the structure of an infinite-dimensional projective variety over $k$, i.e. it is an increasing union of finite-dimensional projective varieties. (Kazhdan and Lusztig work in the case $k=\mathbf{C}$, and their proofs of the results quoted also apply generally in characteristic zero. However, for this paper, it will suffice to know that for classical groups in positive characteristic, these spaces have ind-variety structures and moreover that certain subspaces are algebraic varieties. We will show these results directly.)

We can also generalize the classical partial flag varieties, consisting of the subalgebras of $\mathfrak{g}$ conjugate to a fixed parabolic subalgebra, to the affine context. A parahoric subalgebra of $\mathfrak{g}_{F}$ is a subalgebra containing an Iwahori subalgebra. Let $S$ be a set of simple reflections for the affine Weyl group $\hat{W}$, and for each $I \subseteq S$, let $\hat{W}_{I}$ be the subgroup of $\hat{W}$ generated by $I$. The parahoric subalgebras containing $\hat{\mathfrak{b}}$ are precisely the algebras $\hat{\mathfrak{p}}_{I}=\sum_{w \in \hat{W}_{I}} w \cdot \hat{\mathfrak{b}}$; their stabilizers under the adjoint action are the parahoric subgroups $\hat{P}_{I}=\hat{B} W_{I} \hat{B}$. The map $I \mapsto \hat{\mathfrak{p}}_{I}$ gives a one-to-one correspondence between subsets of $S$ and conjugacy classes of parahoric subalgebras. The set $\hat{\mathcal{P}}_{I}$ of $G(F)$-conjugates of $\hat{\mathfrak{p}}_{I}$ is an infinite-dimensional projective variety which may be identified with the quotient space $G(F) / \hat{P}_{I}$. These are the affine flag manifolds of $G(F)$. The variety $\hat{\mathcal{P}}_{S}$ is just a point, so we will only consider the varieties of proper parahoric subalgebras.

For each (nontrivial) affine flag manifold $\hat{\mathcal{P}}$ and any element $N$ in the Lie algebra $\mathfrak{g}_{F}$, we define $\hat{\mathcal{P}}_{N}$ to be the space of parahoric subalgebras of the specified type containing $N$. This is a closed subvariety of $\hat{\mathcal{P}}$. We shall restrict our attention to the topologically nilpotent (or nil) elements $N$, those elements such that $(\operatorname{ad} N)^{m} \rightarrow 0$ in the power series topology on End $\mathfrak{g}_{F}$ as $m \rightarrow \infty$. More concretely, these are the elements of $\mathfrak{g}_{F}$ which may be conjugated into some $N^{\prime} \in \mathfrak{g}_{A}$ whose image under projection to $\mathfrak{g}$ is nilpotent. Kazhdan and Lusztig have shown in [KL that if $\mathfrak{g}$ is simple, then for $N$ nil, the closed subvariety $\hat{\mathcal{P}}_{N}$ is finite-dimensional (although possibly having infinitely many irreducible components) precisely for $N$ regular semisimple. If we further assume that $N$ is elliptic, i.e. the connected centralizer of $N$ in $G(F)$ is an anisotropic maximal torus, then $\hat{\mathcal{P}}_{N}$ is a projective variety in the usual sense.

The affine fixed point varieties $\hat{\mathcal{P}}_{N}$ are much more complicated than their classical counterparts, and not much is known about their topology. Unlike the $P_{N}$ 's, they need not be rational nor do they necessarily have cell decompositions. For example, even in the low dimensional case $\mathfrak{s p}_{6}(F)$, Bernstein and Kazhdan have found $N$ such that an irreducible component of $\hat{\mathcal{B}}_{N}$ admits a dominant morphism onto an elliptic curve $\mathrm{KL}$. In fact, even the dimensions of these varieties were not known in general until recently, when Bezrukavnikov proved a conjectural formula of Kazhdan and Lusztig.

Lusztig and Smelt have defined $k^{*}$-actions on certain of these fixed point varieties for the special linear groups [LS]. They used these actions to obtain interesting formulas for the Euler characteristics of these subvarieties for the varieties of maximal 
parahoric and Iwahori subalgebras. We have been studying those $\hat{\mathcal{P}}_{N}$ which admit a class of algebraic $k^{*}$-actions which generalize the Lusztig-Smelt actions. In section 2, we define a natural action of the group $Q_{G}^{\prime}=G_{\text {ad }}(k) \times k^{*}$ on the affine flag manifolds of $G(F)$. An algebraic homomorphism $k^{*} \rightarrow Q_{G}^{\prime}$ defines a $k^{*}$-action on $\hat{\mathcal{P}}$ which we call a $Q_{G}^{\prime}$-action. The group $Q_{G}^{\prime}$ also acts on $\mathfrak{g}_{F}$ by $(g, \lambda) \cdot N=\operatorname{Ad}(g) N^{\lambda}$ for $N \in \mathfrak{g}_{F}$, where $N^{\lambda}$ is the image of $N$ under the $k^{*}$-action on $\mathfrak{g}_{F}$ induced by mapping the uniformizing parameter $\pi$ of $F$ to $\lambda \pi$. We say that a $k^{*}$-action $f$ almost commutes with an element $N \in \mathfrak{g}_{F}$ if for some nonzero integer $s, N$ is an eigenvector of $f(\lambda)$ with eigenvalue $\lambda^{s}$ for all $\lambda \in k^{*}$. We show that if $(N, f)$ is an almost commuting pair, then $f$ induces an action on $\hat{\mathcal{P}}_{N}$.

In order to study these actions, we need a more concrete description of our infinite-dimensional varieties, analogous to the identification of the varieties of parabolic subalgebras of $\mathfrak{g}$ with certain varieties of partial flags. Let $\mathfrak{g}$ be a classical Lie algebra, and take the standard representation $\mathfrak{g} \hookrightarrow \mathfrak{g l}(V)$. Set $n$ equal to the dimension of $V$. The varieties of maximal parahoric subalgebras simply consist of certain classes of $A$-lattices in $V_{F}$. The full affine flag manifold $\hat{\mathcal{B}}$ is a space of complete chains of lattices $L_{0} \supset L_{1} \supset \cdots \supset L_{n}$, where $L_{i}$ is of $k$-codimension 1 in $L_{i-1}$ for each $i, L_{n}=\pi L_{0}$, and the lattices $L_{i}$ have certain properties depending on $\mathfrak{g}$. The $\hat{\mathcal{P}}$ for other parahorics are sets of partial lattice chains. Since the Lie algebra action of $\mathfrak{g}_{F}$ on $\hat{\mathcal{P}}$ corresponds to the natural action of $\mathfrak{g}_{F}$ on lattice chains, the variety $\hat{\mathcal{P}}_{N}$ may be identified with those partial lattice chains fixed by $N$. We can now define a $k^{*}$-action on $\hat{\mathcal{P}}_{N}$ by defining a $k^{*}$-action on $V_{F}$ which preserves lattices (and hence lattice chains) of the specified types and then descends to the fixed point subvarieties.

In sections 3 and 4, we identify the affine flag manifolds for $\mathfrak{s l}\left(V_{F}\right)$ and $\mathfrak{s p}\left(V_{F}\right)$ with varieties of lattice chains. We then apply the results of section 2 to obtain complete and concrete descriptions, in terms of the characteristic polynomial, of the nil-elliptic conjugacy classes containing a representative $N$ whose fixed point varieties admit $k^{*}$-actions induced by a $Q_{G}^{\prime}$-action. (For the symplectic algebras, we assume that the characteristic of $k$ is not 2.) Moreover, for each such conjugacy class, we find class representatives in a uniform way for which the lattices fixed by both $N$ and the $k^{*}$-action can be determined explicitly.

These actions are useful and interesting because they enable one to determine the Euler characteristics of the $\hat{\mathcal{P}}_{N}$ 's. By a theorem of Bialynicki-Birula [B-B1], if the multiplicative group $k^{*}$ acts on an algebraic variety $Y$, then the Euler characteristics of $Y$ and $Y^{k^{*}}$ are the same, where $Y^{k^{*}}$ denotes the fixed point set of the action. Therefore, we need only understand the much simpler variety of $k^{*}$ fixed partial lattice chains to calculate the Euler characteristics of the $\hat{\mathcal{P}}_{N}$ 's. In the most favorable cases, including every nil-elliptic conjugacy class of $\mathfrak{s l}\left(V_{F}\right)$ obtained in the above classification, these $k^{*}$-actions only fix diagonal lattices relative to a fixed $k$-basis $e_{1}, \ldots, e_{n}$ of $V$, i.e. lattices of the form $\mathcal{L}_{\mathbf{r}}=A \pi^{r_{1}} e_{1}+\cdots+A \pi^{r_{n}} e_{n}$ for $\mathbf{r} \in \mathbf{Z}^{n}$. This implies that the fixed point set $\left(\hat{\mathcal{P}}_{N}\right)^{k^{*}}$ is discrete and in fact finite, since $N$ is nil-elliptic. We have thus reduced the computation of the Euler characteristic of $\hat{\mathcal{P}}_{N}$ to a purely combinatorial problem of counting lattice chains. For those pairs $(N, f)$ such that $f$ fixes only diagonal lattices, we have discovered simple formulas for the Euler characteristics of $\hat{\mathcal{P}}_{N}$ for each class of parahoric subalgebras as products of binomial coefficients. These formulas can be interpreted as the number of simplices of type corresponding to the parahoric class within a 
certain simplex in an apartment of the affine building of $G$. It is possible to apply similar techniques to the orthogonal algebras; this will be the subject of a future paper.

If an algebraic $k^{*}$-action on a smooth projective variety $Y$ has isolated fixed points, the cells of the Bialynicki-Birula plus decomposition of $Y$ are isomorphic to affine space [B-B2]. The cells are indexed by the fixed points of the action, and the cell corresponding to a fixed point $x$ is $\left\{y \in Y \mid \lim _{\lambda \rightarrow 0} \lambda \cdot y=x\right\}$, where the limit is understood in the sense of B-B3. It is natural to conjecture that when the fixed point set $\left(\hat{\mathcal{P}}_{N}\right)^{k^{*}}$ is finite, the variety $\hat{\mathcal{P}}_{N}$ has such a cell decomposition. For $\mathfrak{s l}(V)$, an argument used by Lusztig and Smelt to obtain this result for Iwahori and maximal parahoric subalgebras applies to show that this is indeed true. In particular, the étale cohomology of $\hat{\mathcal{P}}_{N}$ vanishes in odd degrees in these cases.

These $k^{*}$-actions also have applications to the topology of the classical fixed point varieties. This is in very much the same spirit as Kazhdan and Lusztig's use of nil-elliptic classes in $\mathfrak{g}_{F}$ to study nilpotent classes in $\mathfrak{g}$. Let $X$ denote the variety of conjugates of the maximal parahoric subalgebra $\mathfrak{g}_{A}$. The natural $\mathcal{B}(k)$-fibration $\hat{\mathcal{B}} \stackrel{\rho}{\rightarrow} X$ is given in terms of lattice chains by $\left(L_{0}, \ldots, L_{n}\right) \stackrel{\rho}{\mapsto} L_{0}$. The fibers of this map are the complete flags (of the type corresponding to $\mathfrak{g}$ ) in the $k$-vector space $L_{0} / \pi L_{0}$, and the $N$-fixed lattice chains in the fiber may be interpreted as $\mathcal{B}_{\bar{N}}$, where $\bar{N}$ is the induced nilpotent transformation on $L_{0} / \pi L_{0}$. If $\left(\hat{\mathcal{B}}_{N}\right)^{k^{*}}$ is finite and $L_{0} \in\left(X_{N}\right)^{k^{*}}$, then the Euler characteristic of $\mathcal{B}_{\bar{N}}$ is the number of lattice chains in $\left(\hat{\mathcal{B}}_{N}\right)^{k^{*}}$ lying above $L_{0}$. Similar considerations apply to the partial flag varieties. In section 3, we show that every nilpotent conjugacy class of $\mathfrak{s l}(V)$ may be realized as the class of such an $\bar{N}$. We thus obtain a combinatorial description of the Euler characteristics of the classical fixed point varieties for $\mathfrak{s l}(V)$ without using their explicit cell decomposition or arguments involving induction on unipotent classes. We use a similar technique in section 4 to find combinatorial interpretations of the Euler characteristics of the classical fixed point varieties for $\mathfrak{s p}(V)$ for certain nilpotent classes.

This paper is based on part of my doctoral thesis at the University of Chicago $[\mathrm{S}$. It is a great pleasure to thank my advisor, Robert Kottwitz, for many extremely helpful comments and suggestions. I am also happy to acknowledge the support of a National Science Foundation Graduate Fellowship and a University of Chicago McCormick Fellowship.

\section{2. $k^{*}$-ACTIONS ON FIXED POINT VARIETIES OF AFFINE FLAG MANIFOLDS}

Let $k$ be an algebraically closed field, and let $G=G(k)$ be a semisimple, simply connected, algebraic group over $k$ with Lie algebra $\mathfrak{g}$. Let $F=k((\pi))$ be the field of formal power series in one variable with ring of integers $A=k[[\pi]]$. The local field $F$ is furnished with its usual valuation so that $v(\pi)=1$. We now extend scalars of the group and Lie algebra to the power series field and ring.

Let $p: \mathfrak{g}_{A} \rightarrow \mathfrak{g}$ be the natural projection. An Iwahori (or minimal parahoric) subalgebra of $\mathfrak{g}_{F}$ is a $G(F)$-conjugate of the pullback via $p$ of any Borel subalgebra of $\mathfrak{g}$. The parahoric subalgebras of $\mathfrak{g}_{F}$ are those subalgebras which contain an Iwahori subalgebra. Iwahori and parahoric subgroups of $G(F)$ may be defined in the same manner. Alternatively, a parahoric subgroup is the stabilizer of a parahoric subalgebra under the adjoint action. If $\hat{\mathfrak{p}}$ is a parahoric subalgebra with 
stabilizer $\hat{P}$, then the set of parahoric subalgebras conjugate to $\hat{\mathfrak{p}}$ may be identified with the quotient space $G(F) / \hat{P}$. This space has the natural structure of an infinite-dimensional projective algebraic variety over $k$, i.e. it is an increasing union of finite-dimensional projective varieties. (The results on the variety structure of these spaces are proved by Kazhdan and Lusztig for $k=\mathbf{C}$ (and their proofs work generally in characteristic zero). For classical groups, we may give these spaces the structure of ind-varieties, using their concrete identifications with lattices and lattice chains as explained in section 3. For our computations of the Euler characteristic of certain fixed point subvarieties, it will suffice to know that the spaces in question are indeed algebraic varieties in positive characteristic. Since we will show this directly, in the present paper we will not seek to extend the general results of Kazhdan and Lusztig to characteristic $p$.)

Fix a Borel subalgebra $\mathfrak{b}$ of $\mathfrak{g}$ with $B$ the corresponding Borel subgroup, and let $T$ be a maximal torus of $G$ contained in $B$. The affine Weyl group is a Coxeter group defined by $\hat{W}=N(T(F)) / T(A)$, where $N(T(F))$ is the normalizer of $T(F)$ in $G(F)$. Let $S$ be a set of simple reflections for $\hat{W}$. Then the conjugacy classes of parahoric subalgebras correspond bijectively to the subsets of the simple reflections $S$. Given $I \subseteq S$, let $\hat{W}_{I}$ be the subgroup of $\hat{W}$ generated by $I$. We obtain a standard Iwahori subalgebra $\hat{\mathfrak{b}}$ (with respect to the choice of $\mathfrak{b}$ ) by pulling back $\mathfrak{b}$ to $\mathfrak{g}_{A}$. There is precisely one parahoric subalgebra from each conjugacy class containing $\hat{\mathfrak{b}}$; these are just the subalgebras $\hat{\mathfrak{p}}_{I}=\sum_{w \in \hat{W}_{I}} w \cdot \hat{\mathfrak{b}}$, and with $\hat{B}$ the stabilizer of $\hat{\mathfrak{b}}$, their stabilizers are $\hat{P}_{I}=\hat{B} \hat{W}_{I} \hat{B}$. The corresponding varieties of conjugate subalgebras will be denoted $\hat{\mathcal{P}}_{I}$. If $I \subseteq J$, then $\hat{P}_{I} \subseteq \hat{P}_{J}$, inducing a natural map $\hat{\mathcal{P}}_{I} \rightarrow \hat{\mathcal{P}}_{J}$; this is a fiber bundle with a classical (partial) flag variety as fiber. Note that $\hat{\mathcal{P}}_{S}$ is just a point. From now on, we shall restrict attention to the nontrivial affine flag manifolds or equivalently, to the proper parahoric subalgebras.

We are interested in defining actions of $k^{*}$ on the subvarieties of the affine flag manifolds containing a fixed element $N$ of $\mathfrak{g}_{F}$. As a first step, we will define a natural class of $k^{*}$-actions on the affine flag manifolds themselves. The multiplicative group $k^{*}$ acts by $k$-algebra automorphisms on $F$ via the formula $\lambda \cdot \pi^{m}=\lambda^{m} \pi^{m}$ for all $\lambda \in k^{*}$ and $m \in \mathbf{Z}$. This induces an action of $k^{*}$ on $G(F)$. Since $k^{*}$ preserves the standard Iwahori subgroup $\hat{B}$ and acts trivially on the affine Weyl group, the action descends to the varieties of parahoric subalgebras. Also, $G(F)$ acts on itself by left multiplication. It is now easy to see that the semidirect product $G(F) \rtimes k^{*}$ acts on $G(F)$ by $(g, \lambda) \cdot h=g h^{\lambda}$ where $g, h \in G(F)$ and $\lambda \in k^{*}$ and induces an action on the affine flag manifolds. Since the center $Z(F)$ of $G(F)$ is contained in every parahoric subgroup, we finally obtain an action of $Q_{G}=G_{\text {ad }}(F) \rtimes k^{*}$ on each $\hat{\mathcal{P}}_{I}$, where $G_{\text {ad }}$ is the adjoint form of $G$. We have $G_{\text {ad }}(F)^{k^{*}}=G_{\text {ad }}(k)$, so the group $Q_{G}$ contains the direct product $Q_{G}^{\prime}=G_{\text {ad }}(k) \times k^{*}$. We can now define the class of $k^{*}$-actions which we will study.

Definition. A $k^{*}$-action $f$ on an affine flag manifold for the group $G$ is called a $Q_{G^{-}}^{\prime}$ action if it is obtained from an algebraic homomorphism $k^{*} \stackrel{f}{\rightarrow} Q_{G}^{\prime}$.

Remark. We can similarly define $Q_{G}$-actions, but in this paper, we will restrict attention to $Q_{G}^{\prime}$-actions.

The group $Q_{G}$ also acts on $\mathfrak{g}_{F}$ by $(g, \lambda) \cdot N=\operatorname{Ad}(g) N^{\lambda}$ for $N \in \mathfrak{g}_{F}$. 
Definition. Given $N \in \mathfrak{g}_{F}$, we say that a $k^{*}$-action $f$ almost commutes with $N$ if for some nonzero integer $s, N$ is an eigenvector of $f(\lambda)$ with eigenvalue $\lambda^{s}$, i.e. $f(\lambda) \cdot N=\lambda^{s} N$ for all $\lambda \in k^{*}$. The integer $s$ is called the exponent of the pair $(N, f)$.

This property guarantees that a $Q_{G}$-action $f$ on a conjugacy class of parahoric subalgebras $\hat{\mathcal{P}}$ descends to $\hat{\mathcal{P}}_{N}$, the subvariety of parahorics containing $N$.

Lemma 2.1. Let $\hat{\mathcal{P}}$ be an affine flag manifold, and suppose that $N$ almost commutes with the $Q_{G}$-action $f$. Then $f$ induces an action on $\hat{\mathcal{P}}_{N}$.

Proof. Take $\hat{\mathfrak{p}} \in \hat{\mathcal{P}}_{N}$, and set $f(\lambda)=\left(g(\lambda), \lambda^{p}\right)$ for some $p \in \mathbf{Z}$. (The map of $k^{*}$ into the second factor of $Q_{G}$ is a homomorphism from $k^{*}$ to itself.) Then $N=\lambda^{-s} f(\lambda) \cdot N=\operatorname{Ad} g(\lambda) N^{\lambda^{p}} \in \operatorname{Ad} g(\lambda) \hat{\mathfrak{p}}^{\lambda^{p}}=f(\lambda) \cdot \hat{\mathfrak{p}}$ as desired.

Elements of $\mathfrak{g}_{F}$ which almost commute with a $Q_{G}$-action must satisfy the following condition:

Theorem 2.2. Let $\rho: G \rightarrow G L(V)$ be a representation of $G$, inducing the Lie algebra representation $d \rho: \mathfrak{g}_{F} \rightarrow \mathfrak{g l}\left(V_{F}\right)$. Suppose that $N$ almost commutes with the $Q_{G^{-a c t i o n}} f(\lambda)=\left(g(\lambda), \lambda^{p}\right)$ with exponent $s$. Then the characteristic polynomial $\operatorname{char}_{d \rho(N)}(\mu)$ is a homogeneous polynomial of degree $n=\operatorname{dim} V$ in $\mu$ and $\pi^{q}$ for some rational number $q \in \bigcup_{i=1}^{n} \frac{1}{i} \mathbf{Z}$; if $d \rho(N)$ is not nilpotent, then $p \neq 0$ and $q=s / p$.

Proof. Set $\operatorname{char}_{d \rho(N)}(\mu)=\sum_{i=0}^{n} c_{i} \mu^{n-i}$ for some $c_{i} \in F$. Since $d \rho\left(\operatorname{Ad} g(\lambda) N^{\lambda^{p}}\right)$ and $d \rho\left(N^{\lambda^{p}}\right)$ are $G L\left(V_{F}\right)$-conjugate, we see that $\sum_{i=0}^{n} c_{i}^{\lambda^{p}} \mu^{n-i}=\left(\operatorname{char}_{d \rho(N)}(\mu)\right)^{\lambda^{p}}=$ $\operatorname{char}_{d \rho\left(N^{\lambda^{p}}\right)}(\mu)=\operatorname{char}_{d \rho\left(\lambda^{s} N\right)}(\mu)=\sum_{i=0}^{n} \lambda^{s i} c_{i} \mu^{n-i}$. The claim is trivial if $d \rho(N)$ is nilpotent, so we may assume that $c_{j} \neq 0$ for some $j \geq 1$. The equations $c_{i}^{\lambda^{p}}=\lambda^{s i} c_{i}$ imply that $p v\left(c_{i}\right)=s i$ for each $i$ with $c_{i} \neq 0$; furthermore, $c_{i}$ is homogeneous, i.e. a Laurent series in $\pi$ with at most one nonvanishing term. This shows that $\operatorname{char}_{d \rho(N)}(\mu)$ is homogeneous of degree $n$ in $\mu$ and $\pi^{q}$, where $q=s / p$, and since $s j / p$ is an integer, $q \in \frac{1}{j} \mathbf{Z}$.

Definition. If $N$ is not nilpotent, we call the ratio $s / p$ the loop index of the pair $(N, f)$.

Corollary 2.3. Let $\bar{F}$ be an algebraic closure of $F$. Then all nonzero eigenvalues of $d \rho(N)$ are homogeneous elements of $\bar{F}$ with valuation equal to the loop index.

Proof. By the theorem, $\operatorname{char}_{d \rho(N)}(\mu)=\sum_{i=0}^{n} c_{i}\left(\pi^{q}\right)^{i} \mu^{n-i}$ for some $c_{i} \in k$. The roots of $\operatorname{char}_{d \rho(N)}(\mu)$ in the algebraic closure $\bar{F}$ are just $x \pi^{q}$ where $x \in k$ is a root of the polynomial $\sum_{i=0}^{n} c_{i} \mu^{n-i} \in k[\mu]$.

Recall that $N \in \mathfrak{g}_{F}$ is called $n i l$ (or topologically nilpotent) if $\lim _{m \rightarrow \infty}(\operatorname{ad} N)^{m}=$ 0 . We are primarily interested in defining $k^{*}$-actions on the fixed point variety $\hat{\mathcal{P}}_{N}$ when $N$ is regular semisimple and nil, since in this case, $\hat{\mathcal{P}}_{N}$ is finite-dimensional [KL]. For $N$ of this type in the case of the special linear and symplectic Lie algebras, we will prove a converse statement to theorem 2.2 namely, that if the charactersitic polynomial of a nil, regular semisimple conjugacy class has the form given in theorem [2.2 then we can find an element of the class together with a $Q_{G}$-action almost commuting with it. 
Now assume that $G$ is a classical group, and let $\rho: G \rightarrow G L(V)$ be the standard representation of $G$, inducing the Lie algebra monomorphism $\mathfrak{g} \hookrightarrow \mathfrak{g l}(V)$. In order to define explicit $Q_{G}^{\prime}$-actions when $G$ is a classical group, it will be convenient to consider homomorphisms $k^{*} \rightarrow Q^{\prime} \stackrel{\text { def }}{=} G L(V) \times k^{*}$. Such a homomorphism gives rise to a $Q_{S L(V)}^{\prime}$-action via the projection $G L(V) \rightarrow P G L(V)=P S L(V)$, and if $G$ is not the special linear group, we can obtain a $Q_{G}^{\prime}$-action in a similar way after placing suitable restrictions on the homomorphism.

More generally, if $V$ is an $n$-dimensional vector space over $k, Q^{\prime}$ acts on $V_{F}$ by $k$-linear automorphisms. We call a $k^{*}$-action $f$ on $V_{F}$ a $Q^{\prime}$-action if it comes from an algebraic homomorphism $k^{*} \stackrel{f}{\rightarrow} Q^{\prime}$. For $n \geq 2$, this is equivalent to $f$ mapping $A$-submodules of $V_{F}$ to $A$-submodules and the existence of a $k$-basis $\underline{e}=\left(e_{1}, \ldots, e_{n}\right)$ of $V$ such that every element of the topological spanning set $\underline{\hat{e}}=\left\{\pi^{m} e_{i} \mid m \in \mathbf{Z}, i \in\right.$ $[1, n]\}$ of $V_{F}$ is an eigenvector of $f(\lambda)$ for each $\lambda \in k^{*}$. In fact, the eigenvalue of $\pi^{m} e_{i}$ must have the form $\lambda^{\nu(\cdot, i)}$, where $\nu(\cdot, i): \mathbf{Z} \rightarrow \mathbf{Z}$ is an affine function with slope independent of $i$, i.e. $\nu(m, i)=p m+c_{i}$ for some integers $p$ and $c_{i}[\underline{\mathrm{S}}$, theorem 2.5]. (A specific example of a $k^{*}$-action of this form was first introduced by Lusztig and Smelt $[\mathrm{LS}]$.)

We say that an endomorphism $N \in \mathfrak{g l}\left(V_{F}\right)$ almost commutes with $f$ if for some nonzero integer $s, f(\lambda) N=\lambda^{s} N f(\lambda)$ for all $\lambda \in k^{*}$. The group $Q^{\prime}$ acts on $\mathfrak{g l}\left(V_{F}\right)$, and noting that $f(\lambda) \cdot N=f(\lambda) N f(\lambda)^{-1}$, we see that this is compatible with our previous definition. Fix a basis $\underline{e}$ such that the elements of $\underline{\hat{e}}$ are eigenvectors of the $f(\lambda)$ 's. Let $\left(a_{i j}\right) \in \mathfrak{g l}_{n}(F)$ be the matrix for $N$ determined by $\underline{e}$, and write $a_{i j}=\sum_{l \gg-\infty} a_{i j}^{l} \pi^{l}$ where $a_{i j}^{l} \in k$. Equating the $\pi^{m+l} e_{j}$ terms in $\bar{f}(\lambda) N \pi^{m} e_{i}=$ $\lambda^{s} N f(\lambda) \pi^{m} e_{i}$ for all $m$ and $i$ gives the transformation laws $\nu(m, j)+s=\nu(m+l, i)$ for all $m \in \mathbf{Z}$ and all $i, j$, and $l$ such that $a_{i j}^{l} \neq 0$.

The characteristic polynomial determines the $G L\left(V_{F}\right)$-conjugacy classes of semisimple endomorphisms. We can now characterize the regular semisimple conjugacy classes obtained from almost commuting pairs $(N, f)$ with $f$ a $Q^{\prime}$-action.

Theorem 2.4. Let $\mathcal{C}$ be a regular semisimple conjugacy class in $\mathfrak{g l}\left(V_{F}\right)$. Then there is a representative $N \in \mathcal{C}$ almost commuting with a $Q^{\prime}$-action if and only if the characteristic polynomial chare $(\mu)$ is a homogeneous polynomial of degree $n$ in $\mu$ and $\pi^{q}$ for some nonzero rational number $q \in \frac{1}{n-1} \mathbf{Z} \cup \frac{1}{n} \mathbf{Z}$.

Proof. The forward implication follows just as in theorem 2.2. We need only note that since at most one of the eigenvalues is 0 , either the $\pi^{q n} \mu^{0}$ or the $\pi^{q(n-1)} \mu$ term of the characteristic polynomial has nonzero coefficient, implying that $q \in$ $\frac{1}{n-1} \mathbf{Z} \cup \frac{1}{n} \mathbf{Z}$.

Conversely, assume that for some nonzero $q \in \frac{1}{n-1} \mathbf{Z} \cup \frac{1}{n} \mathbf{Z}$, we have $\operatorname{char}_{\mathfrak{C}}(\mu)=$ $\sum_{i=0}^{n} c_{i}\left(\pi^{q}\right)^{i} \mu^{n-i}$ with each $c_{i} \in k$ and $c_{0}=1$. Define a matrix $N=\left(a_{i j}\right)$ by

$$
a_{i j}= \begin{cases}1 & \text { if } j=i+1, \\ (-1) c_{i} \pi^{i q} & \text { if } j=1, \\ 0 & \text { otherwise. }\end{cases}
$$

Note that if $c_{i} \neq 0$, then $q i \in \mathbf{Z}$. Thus, $a_{i j} \in F$ for each $i$ and $j$.

If the determinant of $N$ is nonzero, set $\nu(m, i)=n m-i n q$; otherwise, set $\nu(m, i)=(n-1) m-i(n-1) q$. It is easy to check that if we define $f(\lambda) \pi^{m} e_{i}=$ $\lambda^{\nu(m, i)} \pi^{m} e_{i}$, where $\left(e_{1}, \ldots, e_{n}\right)$ is the standard basis of $k^{n}$, then $N$ and $f$ almost 
commute with exponent $n q$ (resp. $(n-1) q)$. (The existence of a $Q^{\prime}$-action almost commuting with $N$ is also guaranteed by theorem 2.20 of $[\underline{\mathrm{S}}]$.)

Finally, it follows easily from the definition of the determinant as a sum over the symmetric group $S_{n}$ that $\operatorname{char}_{N}=$ chare. The conjugacy class $\mathcal{C}$ is regular semisimple, so chare has $n$ distinct roots, and since any endomorphism in $\mathfrak{g l}\left(V_{F}\right)$ with $n$ distinct eigenvalues must be diagonalizable, we have $N \in \mathcal{C}$.

It is known that an endomorphism is nil if and only if there exists $N^{\prime} \in \mathfrak{g l}\left(V_{A}\right)$ conjugate to $N$ such that the image of $N^{\prime}$ under projection to $\mathfrak{g l}(V)$ is nilpotent [KL]. It is immediate that all nonzero eigenvalues of a nil endomorphism must have positive valuation. Since a regular semisimple endomorphism with characteristic polynomial as above has eigenvalues of the form $x \pi^{q}$ for some $x \in k$, we obtain the following corollary:

Corollary 2.5. Let $\mathcal{C}$ be a nil, regular semisimple conjugacy class in $\mathfrak{g l}\left(V_{F}\right)$. Then there is a representative $N \in \mathcal{C}$ almost commuting with a $Q^{\prime}$-action if and only if the characteristic polynomial chare $(\mu)$ is a homogeneous polynomial of degree $n$ in $\mu$ and $\pi^{q}$ for some positive rational number $q \in \frac{1}{n-1} \mathbf{Z} \cup \frac{1}{n} \mathbf{Z}$.

\section{3. $k^{*}$-ACTIONS ON FIXED POINT VARIETIES OF AFFINE FLAG MANIFOLDS: THE SPECIAL LINEAR ALGEBRAS}

We will now apply our results on $k^{*}$-actions to the study of fixed point varieties on affine flag manifolds. In this section, we will consider the affine flag manifolds obtained for the root systems $A_{n}$.

Let $V$ be a $k$-vector space of dimension $n \geq 2$. The special linear group $S L(V)$ is the group of linear automorphisms of $V$ which induce the identity on the highest exterior power of $V$, i.e. the volume-preserving linear automorphisms. Its Lie algebra is denoted $\mathfrak{s l}(V)$. Fixing a basis $e_{1}, \ldots, e_{n}$ identifies $S L(V)$ with $S L_{n}(k)$, the semisimple, simply connected, algebraic group over $k$ of type $A_{n-1}$.

Let $\mathfrak{b} \subset \mathfrak{s l}_{n}(k)$ be the standard Borel subalgebra determined by the fixed basis $\underline{e}$, namely the upper triangular matrices in $\mathfrak{s l}_{n}(k)$. The standard Iwahori algebra $\hat{\mathfrak{b}}$ is the pullback of $\mathfrak{b}$ to $\mathfrak{s l}_{n}(A)$. We now define algebras $\hat{\mathfrak{q}}_{i} \subset \mathfrak{s l}_{n}(F)$ for $i \in[0, n-1]$ by

$$
\hat{\mathfrak{q}}_{i}=\mathfrak{s l}_{n}(F) \cap\left(\begin{array}{cc}
i & n-i \\
\pi A & \pi^{-1} A
\end{array}\right) \begin{gathered}
i \\
n-i .
\end{gathered}
$$

The notation means that $\hat{\mathfrak{q}}_{i}$ consists of those matrices in $\mathfrak{s l}_{n}(F)$ of the above form, i.e. the upper right block is an $i \times i$ matrix with entries in $A$ and similarly for the other blocks. Blocks with zero as a dimension are ignored, so $\hat{\mathfrak{q}}_{0}=\mathfrak{s l}_{n}(A)$. These are the maximal (proper) parahoric subalgebras containing $\hat{\mathfrak{b}}$. The stabilizer of $\hat{\mathfrak{q}}_{i}$ is the group $\hat{Q}_{i}$ of matrices in $S L_{n}(F)$ with the same block structure. We can now obtain all parahorics containing $\hat{\mathfrak{b}}$ in terms of the maximal ones: $\hat{\mathfrak{p}}_{I}=\bigcap_{i \notin I} \hat{\mathfrak{q}}_{i}$ with stabilizer $\hat{P}_{I}=\bigcap_{i \notin I} \hat{Q}_{i}$. It is possible to choose simple reflections for the affine Weyl group $\hat{W}$, indexed by the set $[0, n-1]$, such that this notation agrees with the notation of section $2[\underline{\underline{S}}$.

The affine flag manifolds of $S L\left(V_{F}\right)$ may be interpreted as varieties of chains of $A$-lattices in $V_{F}$. Let $\mathcal{L}$ denote the space of all $A$-lattices in $V_{F}$. This set has a natural ind-variety structure. To see this, note that for any lattice $L$, there 
exists a natural number $m$ such that $\pi^{m} V_{A} \subseteq L \subseteq \pi^{-m} V_{A}$. The natural map $L \mapsto L / \pi^{m} V_{A}$ from the set of lattices satisfying this condition for a fixed $m$ to the variety of subspaces of $\pi^{-m} V_{A} / \pi^{m} V_{A}$ is an embedding, identifying the domain as a projective variety. Taking the union over $m$ makes $\mathcal{L}$ into an infinite-dimensional variety. (For classical groups, affine flag manifolds can be interpreted in terms of lattices, and similar arguments show that they are ind-varieties.)

If $L$ is a lattice with basis $f_{1}, \ldots, f_{n}$, then $f_{1} \wedge \cdots \wedge f_{n}=\alpha e_{1} \wedge \cdots \wedge e_{n}$ for some $\alpha \in F^{*}$. The valuation of $\alpha$ is independent of the choice of bases for $L$ and $V$; we call it the valuation of $L$ and denote it by $v(L)$. The space of lattices with fixed valuation $m$ is denoted $\mathcal{L}_{m}$. The group $G L\left(V_{F}\right)$ acts transitively on $\mathcal{L}$, and it is obvious that $T \in G L\left(V_{F}\right)$ maps $\mathcal{L}_{m}$ to $\mathcal{L}_{m+v(\operatorname{det} T)}$. The $S L\left(V_{F}\right)$-orbits of $\mathcal{L}$ are just the varieties $\mathcal{L}_{m}$ for $m \in \mathbf{Z}$.

The actions of $S L\left(V_{F}\right)$ and $F^{*}$ on $\mathcal{L}$ commute, so we see that $\mathcal{L}_{m} \cong \mathcal{L}_{m^{\prime}}$ as an $S L\left(V_{F}\right)$-variety when $m \equiv m^{\prime}(\bmod n)$. (All the $\mathcal{L}_{m}$ 's are isomorphic as varieties, but the $S L\left(V_{F}\right)$-structures are different.) As a result, for each $i \in[0, n-1], S L\left(V_{F}\right)$ acts on the spaces $X_{i}=\left\{\cdots \supset \pi^{-1} L \supset L \supset \pi L \supset \cdots \mid L \in \mathcal{L}_{i}\right\}$. The set $X_{i}$ is just the variety of lattices modulo homothety with valuation congruent to $i$ modulo $n$. This alternate point of view shows that the $\mathcal{L}_{m}$ 's are just special cases of the following more general class of varieties:

Definition. Let $I$ be a nonempty subset of $[0, n-1]$. We call

$$
X_{I}=\left\{\left(L_{i}\right) \mid i \in I(\bmod n), L_{i} \in \mathcal{L}_{i}, L_{i} \supset L_{j} \Longleftrightarrow i<j \text {, and } L_{i+n}=\pi L_{i}\right\}
$$

the space of (partial) lattice chains of type $I$.

The varieties $X_{I}$ are in fact nothing more than the affine flag manifolds for $S L\left(V_{F}\right)$. Note that if $I \subseteq[0, n-1]$ contains 0 , then for any $L \in \mathcal{L}_{0}$, the fiber over $L$ of the projection $X_{I} \rightarrow X_{\{0\}}=\mathcal{L}_{0}$ is isomorphic as an $S L(L / \pi L)$-variety to $\mathcal{F}_{I \backslash\{0\}}$, the variety of flags of type $I \backslash\{0\}$ in $L / \pi L$. Using this observation, we can show that $S L\left(V_{F}\right)$ acts transitively on each $X_{I}$. The automorphism of $\mathbf{Z} / n \mathbf{Z}$ given by $c \mapsto-c$ defines an involution $\alpha$ on the set of coset representatives $[0, n-1]$. The map $I \mapsto \alpha(I)$ is thus a bijective self-map on the set of subsets of $[0, n-1]$. We can find a lattice chain in $X_{I}$ whose stabilizer is $\hat{P}_{\alpha(I)^{c}}$. This proves the following theorem (for more details, see [S, theorem 3.4]):

Theorem 3.1. The varieties $X_{I}$ and $\hat{\mathcal{P}}_{\alpha(I)^{c}}$ are isomorphic for each nonempty subset I of $[0, n-1]$.

In order to apply the results of section 2 on almost commuting pairs to the present context, we will relate regular semisimple conjugacy classes in $\mathfrak{s l}\left(V_{F}\right)$ and $\mathfrak{g l}\left(V_{F}\right)$. Fix an algebraic closure $\bar{F}$ of $F$, and let $F_{s}$ be the separable closure of $F$ in $\bar{F}$. Recall that for the simple root systems, the torsion primes are 2 for $B_{n}, D_{n}$, and $G_{2}, 2$ and 3 for $E_{6}, E_{7}$, and $F_{4}$, and 2,3 , and 5 for $E_{8}$ [SS, I.4.4].

Proposition 3.2. Let $G$ be a simple, simply connected, algebraic group over $k$ with Lie algebra $\mathfrak{g}$, and assume that the characteristic of $k$ is not a torsion prime for $G$. If $N$ is a regular semisimple element of $\mathfrak{g}_{F}$, then the $G(F)$-conjugacy class of $N$ in $\mathfrak{g}_{F}$ is the intersection of $\mathfrak{g}_{F}$ with the $G(\bar{F})$-conjugacy class of $N$ in $\mathfrak{g}_{\bar{F}}$.

Proof. Let $N^{\prime} \in \mathfrak{g}_{F}$ be a $G(\bar{F})$-conjugate of $N$. We claim that $N$ and $N^{\prime}$ are $G\left(F_{s}\right)$-conjugate. Let $Z=Z(\bar{F})$ and $Z^{\prime}=Z^{\prime}(\bar{F})$ be the centralizers of $N$ and $N^{\prime}$ respectively in $G(\bar{F})$. Since the endomorphisms are semisimple, the corresponding 
Lie algebras $\mathfrak{z}$ and $\mathfrak{z}^{\prime}$ are the centralizers of $N$ and $N^{\prime}$ in $\mathfrak{g}_{F}$ [Bo p. 128]. The group $G$ is simply connected and the characteristic of $F$ is not a torsion prime, so by a theorem of Springer and Steinberg, $Z$ and $Z^{\prime}$ are connected [SS, II.3.19]. Since $N$ and $N^{\prime}$ are regular semisimple, they are maximal tori defined over $F$. The tori $Z$ and $Z^{\prime}$ split over $F_{s}$ Bo, III.8.11], hence are conjugate over $G\left(F_{s}\right)$. Choose $h \in G\left(F_{s}\right)$ such that $h Z h^{-1}=Z^{\prime}$, implying that $h \mathfrak{z} h^{-1}=\mathfrak{z}^{\prime}$. But $G(\bar{F})$ conjugacy in the Cartan subalgebra $\mathfrak{z}^{\prime}$ is equivalent to $W$-conjugacy, where $W$ is the Weyl group. Since $W=N\left(Z^{\prime}\left(F_{s}\right)\right) / Z^{\prime}\left(F_{s}\right)$, there exists $h^{\prime} \in N\left(Z^{\prime}\left(F_{s}\right)\right)$ such that $h^{\prime} h N h^{-1} h^{\prime-1}=N^{\prime}$ as desired.

Set $g=h^{\prime} h$. For each $\gamma \in \operatorname{Gal}\left(F_{s} / F\right)$, we have $\gamma(g) N \gamma\left(g^{-1}\right)=N^{\prime}$, so that $g^{-1} \gamma(g)$ is in $Z\left(F_{s}\right)$. The map $\gamma \mapsto \gamma^{-1} \gamma(g)$ is a cocycle with coefficients in the torus $Z\left(F_{s}\right)$; we show that it is a coboundary. The field $F$ is complete with respect to a discrete valuation, and its residue field is algebraically closed. Therefore, the Brauer group of every finite separable extension of $F$ is zero, and this implies that $H^{1}\left(\operatorname{Gal}\left(F_{s} / F\right), T\left(F_{s}\right)\right)=0$ for any torus $T$ defined over $F$ [S1, p. 170]. In particular, $H^{1}\left(\operatorname{Gal}\left(F_{s} / F\right), Z\left(F_{s}\right)\right)=0$. Thus, there exists $z \in Z\left(F_{s}\right)$ such that $z^{-1} \gamma(z)=g^{-1} \gamma(g)$. Note that $\gamma\left(g z^{-1}\right)=g z^{-1}$ for all $\gamma \in \operatorname{Gal}\left(F_{s} / F\right)$, so $g z^{-1} \in$ $G(F)$. The result follows, since $g z^{-1} N z g^{-1}=N^{\prime}$.

Remark. If we assume that char $k=0$, then the statement holds for arbitrary semisimple $N$. In this case, the field $F$ is perfect; moreover, it has cohomological dimension 1 because it is complete with respect to a discrete valuation and has algebraically closed residue field [S2, p. 97]. This implies that $H^{1}(\operatorname{Gal}(\bar{F} / F), L)=0$ for any connected linear group over $F$ [St I.9]. The theorem of Springer and Steinberg again implies that the centralizer $Z$ is connected, and the rest of the proof goes through as above.

A semisimple (resp. regular semisimple) element of $\mathfrak{s l}\left(V_{F}\right)$ is just a (semisimple (resp. regular semisimple) element of $\mathfrak{g l}\left(V_{F}\right)$ with trace zero. Also, two elements of $\mathfrak{s l}\left(V_{\bar{F}}\right)$ are $S L\left(V_{\bar{F}}\right)$-conjugate if and only if they are $G L\left(V_{\bar{F}}\right)$-conjugate. Proposition 3.2 now shows that the regular semisimple conjugacy classes of $\mathfrak{s l}\left(V_{F}\right)$ under the actions of $S L\left(V_{F}\right)$ and $G L\left(V_{F}\right)$ coincide. We thus obtain as an immediate corollary of theorem 2.4 and corollary 2.5 the following classification of regular semisimple conjugacy classes $\mathcal{C}$ in $\mathfrak{s l}\left(V_{F}\right)$ coming from almost commuting pairs $(N, f)$ with $f$ a $Q_{S L(V)}^{\prime}$-action:

Theorem 3.3. Let $\mathcal{C}$ be a regular semisimple (resp. nil regular semisimple) conjugacy class in $\mathfrak{s l}\left(V_{F}\right)$. Then there is a representative $N \in \mathcal{C}$ almost commuting with a $Q_{S L(V)}^{\prime}$-action if and only if the characteristic polynomial chare $(\mu)$ is a homogeneous polynomial of degree $n$ in $\mu$ and $\pi^{q}$ for some nonzero (resp. positive) rational number $q \in \frac{1}{n-1} \mathbf{Z} \cup \frac{1}{n} \mathbf{Z}$.

Note that $c_{1}=\operatorname{tr} \mathcal{C}=0$ in $\operatorname{char}_{\mathcal{C}}(\mu)=\sum_{i=0}^{n} c_{i} \pi^{q i} \mu^{n-i}$, so the standard representative for a conjugacy class given in the proof of theorem 2.4 lies in $\mathfrak{s l}\left(V_{F}\right)$.

We are primarily interested in defining $k^{*}$-actions on the fixed point varieties $\hat{\mathcal{P}}_{N}$ when they are algebraic varieties. Recall that a torus $T$ over $F$ is called elliptic if no nontrivial cocharacter of $T$ is defined over $F$. We say that a regular semisimple element $N \in \mathfrak{s l}\left(V_{F}\right)$ is elliptic if its centralizer $Z_{S L\left(V_{F}\right)}(N)$ in $S L\left(V_{F}\right)$ is an elliptic torus. Suppose that $N \in \mathfrak{s l}\left(V_{F}\right)$ is nil. By a theorem of Kazhdan and Lusztig [KL], $\operatorname{dim} \hat{\mathcal{P}}_{N}<\infty$ if and only if $N$ is regular semisimple. Furthermore, $\hat{\mathcal{P}}_{N}$ is an 
algebraic variety, i.e. has a finite number of irreducible components precisely for $N$ elliptic. (For positive characteristic, we note that the fixed point subvarieties considered below are algebraic varieties for an arbitrary algebraically closed field; this follows from [LS.)

It is known that a regular semisimple element $N$ of $\mathfrak{s l}\left(V_{F}\right)$ is elliptic if and only if $\operatorname{char}_{N}(\mu) \in F[\mu]$ is an irreducible, separable polynomial $[\underline{\mathrm{S}}$, proposition 3.10]. If $F$ has characteristic zero, then for each positive integer $m$, the field $F$ has a unique extension of degree $m$ in $\bar{F}$; it is generated by an $m$ th root of $\pi$. If we call this cyclic extension $F\left(\pi^{1 / m}\right)$, we have $\bar{F}=\bigcup_{m=1}^{\infty} F\left(\pi^{1 / m}\right)$. In characteristic $p$, the structure of $\bar{F}$ and the separable closure $F_{s}$ is much more complicated. However, the previous theorem guarantees that any elliptic, regular semisimple $N$ almost commuting with a $Q_{S L(V)}^{\prime}$-action has eigenvalues lying in $\bigcup_{p \nmid m} F\left(\pi^{1 / m}\right) \subset F_{s}$. Therefore, if $N$ is nil-elliptic, the splitting field of $\operatorname{char}_{N}$ is $F\left(\pi^{1 / n}\right)$ and $n$ can not be a multiple of the characteristic. By corollary 2.3 each eigenvalue is homogeneous of valuation $q=s / n>0$ where $s$ is relatively prime to $n$. Since $\pi^{q i} \notin F$ for $i \in[1, n-1]$, we have $\operatorname{char}_{N}(\mu)=\mu^{n}-b \pi^{s}$. Let $a \in k$ be some $n$th root of $b$. Then the roots of $\operatorname{char}_{N}$ over $\bar{F}$ are $\left\{a \zeta \pi^{q}: \zeta\right.$ an $n$th root of unity in $\left.k\right\}$. These are distinct if and only if $a \neq 0$ and the characteristic of $k$ is either 0 or relatively prime to $n$. This can be rewritten more simply as $\left(\operatorname{char}^{\prime} k, n\right)=1$, where $\operatorname{char}^{\prime} k$ denotes the characteristic exponent of $k$. (Recall that the characteristic exponent of a field is 1 if the field has characteristic 0 and equals the characteristic otherwise ([B2, 5.1.5]). Summing up, we have:

Proposition 3.4. The nil-elliptic conjugacy classes in $\mathfrak{s l}\left(V_{F}\right)$ obtained from almost commuting pairs $(N, f)$ with $f$ a $Q_{S L(V)}^{\prime}$-action are precisely those with chare $(\mu)=$ $\mu^{n}-b \pi^{s}$ where $b \in k^{*}, s>0,(s, n)=1$, and $\left(\operatorname{char}^{\prime} k, n\right)=1$. Fixing a basis $\underline{e}$ for $V$, we have the standard matrix representative

$$
N_{b s}=\left(\begin{array}{cccccc}
0 & 1 & 0 & 0 & \ldots & 0 \\
0 & 0 & 1 & 0 & \ldots & 0 \\
\vdots & \vdots & \ddots & \ddots & & \vdots \\
\vdots & \vdots & \ddots & \ddots & 0 & 1 \\
b \pi^{s} & 0 & 0 & \ldots & 0 & 0
\end{array}\right)
$$

Each $N_{b s}$ almost commutes with exponent $s$ with the $Q^{\prime}$-action $f$, diagonal with respect to $\underline{e}$, given by $\nu(m, i)=n m-i$ s.

Note that the field automorphism of $F$ given by $\pi \mapsto b^{1 / s} \pi$ (where $b^{1 / s}$ is a fixed $s$ th root of $b$ ) induces an automorphism of $\mathfrak{g}_{F}$ sending $N_{1 s}$ to $N_{b s}$. Accordingly, $\hat{\mathcal{P}}_{N_{b s}}$ is isomorphic to $\hat{\mathcal{P}}_{N_{1 s}}$, and we need only consider the case $b=1$. For simplicity of notation, we set $N_{s} \stackrel{\text { def }}{=} N_{1 s}$.

Remark. Lusztig and Smelt used different representatives for these nil-elliptic conjugacy classes and almost commuting actions [LS].

For the remainder of the section, we assume that $\left(n, \operatorname{char}^{\prime} k\right)=1$ and fix a basis $\underline{e}$ of $V$. By the Iwasawa decomposition for $G L_{n}(F)$, any lattice $L$ has a basis $\underline{z}$ such that $z_{\underline{e}}$ is upper triangular. Indeed, $L$ has a unique basis of the form $z_{j}=$ $\pi^{r_{j}} e_{j}+\sum_{i<j} z_{i j} e_{i}$ for $j \in[1, n]$, where $r_{j} \in \mathbf{Z}$ and $z_{i j} \in F$ is a terminating Laurent series with all nonzero terms of degree strictly smaller than $r_{i}$. The diagonal lattices 
(with respect to $\underline{e}$ ) are the lattices $\Lambda_{\mathbf{r}}=A \pi^{r_{1}} e_{1}+\cdots+A \pi^{r_{n}} e_{n}$ for $\mathbf{r} \in \mathbf{Z}^{n}$; they form a countable closed subset $\mathcal{D}$ of $\mathcal{L}$. Note that $\mathcal{D}_{m} \stackrel{\text { def }}{=} \mathcal{D} \cap \mathcal{L}_{m}=\left\{\Lambda_{\mathbf{r}} \mid \sum_{i=1}^{n} r_{i}=m\right.$. We call the set of lattices chains in $X_{I}$ composed of diagonal lattices $\mathcal{D}_{I}$.

It is obvious that any $Q^{\prime}$-action diagonal with respect to $\underline{e}$ fixes every lattice in $\mathcal{D}$. The converse is not true in general, but it does hold for the $Q^{\prime}$-actions defined in the above theorem.

Lemma 3.5. Let $f$ be the $Q^{\prime}$-action almost commuting with $N_{s}$ in proposition 3.4. The fixed point set of $f$ on $\mathcal{L}$ is $\mathcal{D}$.

Proof. Let $L$ be a lattice fixed by $f$, and choose a standard basis $\underline{z}$ for $L$ as described above. We will show that the off-diagonal components of $z_{j}$ are zero by induction on $j$. The claim is vacuous for $j=1$. Suppose that $j>1$. Since $f(\lambda) z_{j} \in L$, we have

$$
f(\lambda) z_{j}=\sum_{i^{\prime}=1}^{n} a_{i^{\prime}} z_{i^{\prime}}
$$

for some elements $a_{i^{\prime}} \in A$. It is immediate that $a_{i^{\prime}}=0$ for $i^{\prime}>j$; if $i>j$ is the largest index such that $a_{i} \neq 0$, then equating the coefficients of $e_{i}$ in this expression gives $a_{i}=0$, a contradiction. By comparing the $e_{j}$ terms, we now get $\lambda^{\nu\left(r_{j}, j\right)} \pi^{r_{j}}=a_{j} \pi^{r_{j}}$, i.e. $a_{j}=\lambda^{\nu\left(r_{j}, j\right)}$. If $i<j$, then the $e_{i}$ component of $z_{i^{\prime}}$ vanishes unless $i^{\prime}=i$ or $i^{\prime}=j$ by inductive hypothesis. Writing $z_{i j}=\sum_{m \gg-\infty}^{r_{i}-1} z_{i j}^{m} \pi^{m}$ and equating the $e_{i}$ terms on each side of (3-1), we have

$$
\sum_{m \gg-\infty}^{r_{i}-1} \lambda^{\nu(m, i)} z_{i j}^{m} \pi^{m}=a_{i} \pi^{r_{i}}+\lambda^{\nu\left(r_{j}, j\right)} \sum_{m \gg-\infty}^{r_{i}-1} z_{i j}^{m} \pi^{m} .
$$

Therefore,

$$
a_{i}=\pi^{-r_{i}} \sum_{m \gg-\infty}^{r_{i}-1}\left(\lambda^{\nu(m, i)}-\lambda^{\nu\left(r_{j}, j\right)}\right) z_{i j}^{m} \pi^{m} \in A .
$$

Since $m-r_{i}<0,\left(\lambda^{\nu(m, i)}-\lambda^{\nu\left(r_{j}, j\right)}\right) z_{i j}^{m}=0$ for each $m$. If $\lambda^{\nu(m, i)}-\lambda^{\nu\left(r_{j}, j\right)}=0$, then $n\left(r_{j}-m\right)=(j-i) s$, a contradiction since $(s, n)=1$ and $n$ does not divide $j-i \in[1, n-1]$. Thus, $z_{i j}^{m}=0$ for all $m$, i.e. $z_{j}=\pi^{r_{j}} e_{j}$.

Remarks. 1. Using a similar argument, it is possible to show that if $f$ is a diagonal $Q^{\prime}$-action almost commuting with a nonnilpotent element $N \in \mathfrak{g l}_{n}(F)$ and if $(N, f)$ has loop index $s / n$ with $(s, n)=1$, then the fixed point set of $f$ on $\mathcal{L}$ is $\mathcal{D}[\mathbf{S}$. proposition 3.12].

2. It is an immediate consequence that $\mathcal{D}$ is closed in $\mathcal{L}$.

For any nil-elliptic $N \in \mathfrak{s l}\left(V_{F}\right), \mathcal{D} \cap \mathcal{L}_{m, N}$ is an algebraic variety and hence has a finite number of components. Since $\mathcal{D}$ is discrete, the proposition then shows that the fixed point sets $\left(\hat{\mathcal{P}}_{N_{s}}\right)^{k^{*}}$ for the $k^{*}$-action $f$ of proposition 3.4 are all finite. A theorem of Bialynicki-Birula [B-B1] implies that $\chi\left(\hat{\mathcal{P}}_{N_{s}}\right)=\chi\left(\left(\hat{\mathcal{P}}_{N_{s}}\right)^{k^{*}}\right)$, so the calculation of these Euler characteristics has been reduced to a problem in combinatorics.

We can decompose the computation into two complementary parts. Take $I \subsetneq$ $[0, n-1]$ and let $J=[0, n-1] \backslash\{m\}$ be a maximal proper subset of $[0, n-1]$ 
containing $I$. The fiber bundle $\hat{\mathcal{P}}_{I} \stackrel{\rho}{\rightarrow} \hat{\mathcal{P}}_{J}$ with fiber $\mathcal{P}$ induces a map $\hat{\mathcal{P}}_{I, N} \rightarrow \hat{\mathcal{P}}_{J, N}$. Upon passing to points fixed by $f$, we see that

$$
\chi\left(\hat{\mathcal{P}}_{I, N}\right)=\chi\left(\left(\hat{\mathcal{P}}_{I, N}\right)^{k^{*}}\right)=\sum_{z \in\left(\hat{\mathcal{P}}_{J, N}\right)^{k^{*}}}\left|\left(\rho^{-1}(z)\right)^{k^{*}}\right| .
$$

Suppose $z \in\left(\hat{\mathcal{P}}_{J, N}\right)^{k^{*}}$ corresponds to the lattice $L \in \mathcal{L}_{m}$. Let $\bar{N} \in \mathfrak{s l}(L / \pi L)$ be the nilpotent endomorphism induced by $N$. The summand may then be interpreted as the Euler characteristic of $\mathcal{P}_{\bar{N}}$, a fixed point variety on a classical flag manifold. We can thus approach the problem by examining the fixed points in the base and fibers of $\rho$ separately.

We will first determine the fixed point sets $\left(\mathcal{L}_{m, N_{s}}\right)^{k^{*}}$. Let $\Lambda$ be a lattice of valuation $m$ fixed by $f$ and $N_{s}$. Then $\Lambda$ must equal $\Lambda_{\mathbf{r}}$ for some $\mathbf{r} \in \mathbf{Z}^{n}$ with $\sum_{i=1}^{n} r_{i}=m$. The inclusion $N_{s} \Lambda \subset \Lambda$ implies that $\pi^{r_{i}} e_{i-1} \in A \pi^{r_{i-1}} e_{i-1}$ for $i \in[2, n]$ and $\pi^{r_{1}+s} \in A \pi^{n}$. Thus, $\left(\mathcal{L}_{m, N_{s}}\right)^{k^{*}}=\left\{\Lambda_{\mathbf{r}} \mid \mathbf{r} \in R_{s m}\right\}$ where

$$
R_{s m}=\left\{\mathbf{r} \in \mathbf{Z}^{n} \mid \sum_{i=1}^{n} r_{i}=m \text { and } r_{1} \leq r_{2} \leq \cdots \leq r_{n} \leq r_{1}+s\right\} .
$$

To determine the size of $R_{s m}$, we will use an argument due to Lusztig and Smelt [LS]. For $i \in[1, n]$, set $\zeta_{i}=\delta_{i 1} s$ and define a function $\Theta_{s}: \mathbf{Z} \rightarrow \mathbf{Z}$ by $\Theta_{s}(l)=\sum_{i=1}^{n}(l+i)\left(r_{i}-r_{i-1}+\zeta_{i}\right)$ (where we view $\mathbf{r}$ as a periodic sequence indexed by $\mathbf{Z})$. We have

$$
\begin{aligned}
\Theta_{s}(l)=\Theta_{s}(0)+l \sum_{i=0}^{n-1}\left(r_{i+1}-r_{i}+\zeta_{i}\right) & =n r_{n}-\sum_{i=1}^{n} r_{i}+s+l s \\
& =-m+(l+1) s+n r_{n} \\
& \equiv-m+(l+1) s \quad(\bmod n) .
\end{aligned}
$$

Hence, $\Theta_{s}$ induces a map $\mathbf{Z} / n \mathbf{Z} \rightarrow \mathbf{Z} / n \mathbf{Z}$, and it is in fact a bijection because $s$ and $n$ are relatively prime. Set $C_{s}^{n}=\left\{\mathbf{c} \in \mathbf{N}^{n} \mid \sum_{i=1}^{n} c_{i}=s\right\}$ and $C_{s m}^{n}=\left\{\mathbf{c} \in C_{s}^{n} \mid\right.$ $\left.\sum_{i=1}^{n}(l+i) c_{i} \equiv-m+(l+1) s(\bmod n)\right\}$. Usually the superscript will be suppressed from the notation. Just as in (3-4), the congruence will hold for each integer if it holds for a single $l$. The group $\mathbf{Z} / n \mathbf{Z}$ acts on $C_{s}$ via cyclic permutations of the coordinates; let $\tilde{C}_{s}$ denote the set of orbits.

Lemma 3.6. There are bijections $R_{s m} \stackrel{\phi}{\rightarrow} C_{s m} \stackrel{\psi}{\rightarrow} \tilde{C}_{s}$.

Proof. Set $\phi(\mathbf{r})_{i}=r_{i}-r_{i-1}+\zeta_{i}$. If $\phi(\mathbf{r})=\phi\left(\mathbf{r}^{\prime}\right)$, then equality of the last $n-1$ components implies that $\mathbf{r}^{\prime}=\mathbf{r}+t \mathbf{1}$ where $t \in \mathbf{Z}$ and $\mathbf{1}$ is the constant $n$-tuple $(1, \ldots, 1)$. The sums of the coefficients are equal, so $t=0$. Thus, $\phi$ is injective. Now take $\mathbf{c} \in C_{s m}$. Set

$$
r_{n}=\frac{m-s+\sum_{i=1}^{n} i c_{i}}{n} \text { and } r_{j}=r_{n}-\left(c_{j+1}+\cdots+c_{n}\right) \text { for } j \in[1, n-1] .
$$

These are integers by definition of $C_{s m}$. We have

$$
\sum_{i=1}^{n} r_{i}=n r_{n}-\sum_{i=1}^{n}(i-1) c_{i}=m-s+\sum_{i=1}^{n} c_{i}=m .
$$

This proves surjectivity. 
Let $\psi$ be the restriction of the orbit map to $C_{s m}$. The $n$-cycle $\tau=(1,2, \ldots, n)$ generates the $\mathbf{Z} / n \mathbf{Z}$ action. Note that for any $\mathbf{c} \in C_{s}$,

$$
\sum_{i=1}^{n} i(\tau \cdot \mathbf{c})_{i}=\sum_{i=1}^{n}(1+i) c_{i}-n c_{n} \equiv s+\sum_{i=1}^{n} i c_{i} \quad(\bmod n) .
$$

This means that within the orbit of c, the sum runs through the residue classes $\bmod n$, i.e. precisely one representative lies in $C_{s m}$. Therefore, $\psi$ is bijective.

Corollary 3.7. For any $m \in \mathbf{Z}$,

$$
\chi\left(\mathcal{L}_{m, N_{s}}\right)=\frac{(n+s-1) !}{n ! s !} .
$$

Proof. The proof of the lemma shows that $\mathbf{Z} / n \mathbf{Z}$ acts freely on $C_{s}$. Consequently, $\chi\left(\mathcal{L}_{m, N_{s}}\right)=\left|R_{s m}\right|=\left|\tilde{C}_{s}\right|=\left|C_{s}\right| / n$. It now suffices to recall the well known fact that $\left|C_{s}\right|=\left(\begin{array}{c}n+s-1 \\ n-1\end{array}\right)=\frac{(n+s-1) !}{(n-1) ! s !}$; it is just the number of ways of putting $s$ indistinguishable balls into $n$ boxes.

We have just shown that $\left|\tilde{C}_{s}^{n}\right|=\frac{(n+s-1) !}{n ! s !}$. This expression is symmetrical in $n$ and $s$, so we have a bijection $\tilde{C}_{s} \stackrel{\sim}{\rightarrow} \tilde{C}_{n}^{s}$. We can describe this bijection explicitly. The orbit $\mathbf{c} \in \tilde{C}_{s}^{n}$ may be interpreted as an arrangement of $s$ indistinguishable balls in $n$ boxes placed around a circle. Viewing a box as two boundary walls, we see that this is just a configuration of $s$ balls and $n$ walls up to cyclic permutation of the walls. However, because $n$ and $s$ are relatively prime, this is equivalent to an arrangement of the $n+s$ objects up to cyclic permutation. Let a cell be a "dual box", where the boundaries are now balls and the contents of the cell are walls. The analogous argument now gives a way of placing $n$ walls in $s$ cells around a circle and hence defines the desired bijection. More concretely, number the boxes and walls clockwise around the circle so that the $(t-1)$ st and $t$ th walls bound the $t$ th box. Label the balls and cells similarly, starting with the first ball counterclockwise of wall 1. Letting $B_{k}$ denote the set of walls contained in the $k$ th cell, we have $b_{k}=\left|B_{k}\right|$. The cell numbering guarantees that for walls $t, t^{\prime}$ within a given cell, $t<t^{\prime}$ if and only if the path from $t$ to $t^{\prime}$ staying in the cell runs clockwise.

We now turn to lattice chains of type $J^{\prime}$ where $J^{\prime}$ is a nonempty subset of $[0, n-1]$. Let $l$ be the number of elements in $J^{\prime}$. Choose $m \in J^{\prime}$ and let $J=\left\{j_{1}<\cdots<j_{l}=\right.$ $n\} \subseteq[1, n]$ be the unique set such that $J^{\prime}=m+\left((J \backslash\{n\}) \cup\left\{j_{0}=0\right\}\right)(\bmod n)$. Set $p_{i}=j_{i}-j_{i-1}$. A chain in $X_{J^{\prime}}$ is uniquely determined by its component lattices in $\mathcal{L}_{m+j}$ for $j \in J$. Thus, $\left(X_{J^{\prime}, N_{s}}\right)^{k^{*}}=\left\{\left(\Lambda_{\mathbf{r}^{j}}\right)_{j \in J} \mid\left(\mathbf{r}^{0}, \ldots, \mathbf{r}^{j_{l}}\right) \in Y_{J s m}\right\}$ where

$$
Y_{J s m}=\left\{\left(\mathbf{r}^{0}, \ldots, \mathbf{r}^{j_{l}}\right) \mid \mathbf{r}^{j_{i}} \in R_{s, m+j_{i}} \text { and } \mathbf{r}^{0} \leq \mathbf{r}^{j_{1}} \leq \cdots \leq \mathbf{r}^{j_{l-1}} \leq \mathbf{r}^{n}=\mathbf{r}^{0}+\mathbf{1}\right\} .
$$

The partial order on vectors is defined componentwise.

Fix $\mathbf{r} \in R_{s m}$ and let $Y_{J s m}(\mathbf{r})$ denote those chains in $Y_{J s m}$ with $\mathbf{r}^{0}=\mathbf{r}$. Given any chain $\hat{\mathbf{r}}$ in this fiber, $r_{t} \leq r_{t}^{j_{1}} \leq \cdots \leq r_{t}^{j_{l}}=r_{t}+1$ for each $t \in[1, n]$. Hence, there exists a unique $\sigma(t)=j_{i} \in J$ such that $r_{t}^{j_{i-1}}=r_{t}$ and $r_{t}^{j_{i}}=r_{t}+1$. The map $\sigma:[1, n] \rightarrow J$ partitions $[1, n]$ into subsets $\sigma^{-1}\left(j_{i}\right)$ of size $p_{i}$ and uniquely determines $\hat{\mathbf{r}}$. Let $\mathbf{b} \in C_{n}^{s}$ be a representative of the element of $\tilde{C}_{n}^{s}$ corresponding to $\mathbf{r}$ through the bijections $R_{s m} \rightarrow \tilde{C}_{s} \rightarrow \tilde{C}_{n}^{s}$. We define a set of functions

$$
Q_{J s}^{\prime}(\mathbf{b})=\left\{\sigma:[1, n] \rightarrow J|| \sigma^{-1}(j) \mid=p_{j} \text { and } \sigma \text { is nonincreasing on each } B_{k}\right\} .
$$


Note that although this set depends on a choice of labeling of the cells $B_{k}$, any other labeling (not just those obtained through cyclic permutations) or even any decomposition of $[1, n]$ into $s$ disjoint subsets of sizes $b_{k}$ gives a set of the same size.

Proposition 3.8. The map $\hat{\mathbf{r}} \mapsto \sigma$ defines a bijection $Y_{J s m}(\mathbf{r}) \stackrel{\sim}{\rightarrow} Q_{J s}^{\prime}(\mathbf{b})$.

Proof. Take $\hat{\mathbf{r}} \in Y_{J s m}$. Note that $t-1$ and $t$ are in $B_{k}$ if and only if $c_{t}=0 ; c_{t} \neq 0$ means that box $t$ contains a ball, separating walls $t-1$ and $t$ into different cells. Suppose that $t-1$ and $t$ are in $B_{k}$, but $\sigma(t)>\sigma(t-1)=j_{i}$. Then $r_{t}^{j_{i}}=r_{t}$ and $r_{t-1}^{j_{i}}=r_{t-1}+1$, implying that $c_{t}^{j_{i}}=r_{t}-\left(r_{t-1}+1\right)=c_{t}-1=-1$, a contradiction. Moreover, for each $j \in J, \sum_{t=1}^{n} r_{t}^{j}=m+j$, so $r_{t}^{j_{i-1}}<r_{t}^{j}$ for exactly $p_{i}$ elements of $[1, n]$. Thus, the map sends $Y_{J s m}$ into $Q_{J s}^{\prime}(\mathbf{b})$.

The map is clearly injective. Take $\sigma \in Q_{J s}^{\prime}(\mathbf{b})$ and define $r^{j_{i}}$ recursively by

$$
r_{t}^{j_{i}}= \begin{cases}r_{t}^{j_{i-1}}+1 & \text { if } t \in \sigma^{-1}\left(j_{i}\right), \\ r_{t}^{j_{i-1}} & \text { if } t \notin \sigma^{-1}\left(j_{i}\right) .\end{cases}
$$

We will show that $\mathbf{r}^{j_{i}} \in R_{s, m+j_{i}}$ by induction on $i$. This is trivial for $i=0$. Now suppose that $i>0$ and that the claim is true for indices smaller than $i$. In particular, $\mathbf{c}^{j_{i^{\prime}}}=\phi\left(\mathbf{r}^{j_{i^{\prime}}}\right)$ is well-defined for all $i^{\prime}<i$. The coordinates of the $n$-tuple $\mathbf{r}^{j_{i}}$ sum to $m+j_{i}$ because $\sum_{t=1}^{n} r_{t}^{j_{i}}=\sum_{t=1}^{n} r_{t}^{j_{i-1}}+\left|\sigma^{-1}\left(j_{i}\right)\right|=m+j_{i-1}+p_{i}=j_{i}$. Also, observe that if $r_{t}^{j_{i}}+\zeta_{t}<r_{t-1}^{j_{i}}$, then $r_{t}^{j_{i-1}}+\zeta_{t}=r_{t-1}^{j_{i-1}}$, i.e. $c_{t}^{j_{i-1}}=0$. Thus, $\sigma(t-1)=j_{i}$, and since $c_{t}^{j_{i^{\prime}}}$ only decreases where $r_{t-1}^{j_{i^{\prime}}}$ increases, namely at the $i$ th step, $c_{t}^{j_{i^{\prime}}}=0$ for each $i^{\prime}<i$. In particular, $c_{t}=0$. But this means that $t$ and $t-1$ are in the same cell, so $j_{i}=\sigma(t-1) \geq \sigma(t)$. This is a contradiction; if $\sigma(t-1)=\sigma(t)$, then $0=r_{t}^{j_{i-1}}-r_{t-1}^{j_{i-1}}+\zeta_{t}=r_{t}^{j_{i}}-r_{t-1}^{j_{i}}+\zeta_{t}<0$ and if $\sigma(t-1)>\sigma(t)=j_{i^{\prime}}$, then $c_{t}^{j_{i^{\prime}}}=1$ for some $i^{\prime}<i$. This proves surjectivity.

Definition. Let $\mathbf{d}=\left(d_{0}, d_{1}, \ldots, d_{l}\right)$ be a nondecreasing sequence and $t$ a positive integer. We call a matrix $\left(q_{i k}\right) \in M_{l t}(\mathbf{N})$ a $(\mathbf{d}, t)$-intersection matrix if $\sum_{k=1}^{t} q_{i k}=$ $d_{i}-d_{i-1}$ for $i \in[1, l]$. We denote the set of $(\mathbf{d}, t)$-intersection matrices by $Q_{\mathbf{d} t}$. If $\mathbf{d}$ is strictly increasing, then the $d_{i}-d_{i-1}$ 's are determined by $D=\left\{d_{0}, \ldots, d_{l}\right\}$. In this case, $Q_{D t} \stackrel{\text { def }}{=} Q_{\mathbf{d} t}$ is called the set of $(D, t)$-intersection matrices. For any $\mathbf{b} \in C_{d_{l}-d_{0}}^{t}$, let $Q_{\mathbf{d} t}(\mathbf{b}) \subseteq Q_{\mathbf{d} t}$ be the set of $(\mathbf{d}, t)$-intersection matrices with $\sum_{i=1}^{l} q_{i k}=b_{k}$ for each $k \in[1, t]$. The group $\mathbf{Z} / t \mathbf{Z}$ acts freely on $Q_{\mathbf{d} t}$ by cyclic permutation of the columns; we call the set of orbits $\tilde{Q}_{\mathbf{d} t}$.

\section{Lemma 3.9.}

$$
\left|Q_{\mathbf{d} t}\right|=\prod_{i=1}^{l}\left(\begin{array}{c}
s+d_{i}-d_{i-1}-1 \\
d_{i}-d_{i-1}
\end{array}\right) .
$$

Proof. The $i$ th row of a $(\mathbf{d}, t)$-intersection matrix is just an element of $C_{d_{i}-d_{i-1}}^{t}$, so we have a bijection $Q_{\mathbf{d} t} \rightarrow \prod_{i=1}^{l} C_{d_{i}-d_{i-1}}^{t}$. The result follows, since $\left|C_{d_{i}-d_{i-1}}^{t}\right|=$ $\left(\begin{array}{c}t+d_{i}-d_{i-1}-1 \\ d_{i}-d_{i-1}\end{array}\right)$ by the proof of corollary 3.7 .

We will describe the Euler characteristics of $X_{J, N_{s}}$ in terms of $(J, s)$-intersection matrices. Define a map $Q_{J s}^{\prime}(\mathbf{b}) \rightarrow Q_{J s}(\mathbf{b})$ by $\sigma \mapsto\left(\left|\sigma^{-1}\left(j_{i}\right) \cap B_{k}\right|\right)$. (This is the motivation for the terminology.)

Proposition 3.10. The map $Q_{J s}^{\prime}(\mathbf{b}) \stackrel{\sim}{\rightarrow} Q_{J s}(\mathbf{b})$ is a bijection. 
Proof. Fix an intersection matrix $Q=\left(q_{i k}\right) \in Q_{J s}(\mathbf{b})$, and suppose $\sigma$ is mapped to $Q$. The function $\sigma$ is nonincreasing on $B_{k}$, so the top $q_{1 k}$ elements of $B_{k}$ are in $\sigma^{-1}(1)$, the next highest $q_{2 k}$ are in $\sigma^{-1}(2)$, and so on. Thus, $\sigma$ is completely determined by its intersection matrix. Conversely, this procedure clearly defines $\sigma \in Q_{J s}^{\prime}(\mathbf{b})$ mapping to $Q$.

We can apply this proposition to obtain a combinatorial description of the Euler characteristic of $\mathcal{P}_{N}$ for any classical partial flag variety $\mathcal{P}$ and any nilpotent $N \in$ $\mathfrak{s l}_{n}(k)$. Nilpotent conjugacy classes in $\mathfrak{s l}_{n}(k)$ are determined by the Jordan blocks of $N$, in other words, by a partition of $n$, say $n=n_{1}+\cdots+n_{t}$ where each $n_{i}$ is positive.

Theorem 3.11. Assume that $\left(\right.$ char $\left.^{\prime} k, n\right)=1$. Let $N \in \mathfrak{s l}_{n}(k)$ be a nilpotent element in the conjugacy class corresponding to the partition $n=n_{1}+\cdots+n_{t}$. Let $s$ be any integer greater than $t$ and relatively prime to $n$. Define $\mathbf{b} \in C_{n}^{s}$ by $b_{i}=n_{i}$ for $i \in[1, t]$ and 0 otherwise. Then for each $I \subseteq[1, n-1], \chi\left(\mathcal{F}_{I, N}\right)=\left|Q_{I \cup\{n\}, s}(\mathbf{b})\right|$. In particular, $\chi\left(\mathcal{B}_{N}\right)=\frac{n !}{n_{1} ! \cdots n_{t} !}$.

Proof. Let $\mathbf{r} \in R_{s 0}$ correspond to the orbit of $\mathbf{b}$. Since the fiber of the fixed point set over $\mathbf{r}$ corresponds bijectively to $Y_{I q \cup\{n\}, s 0}(\mathbf{r}) \stackrel{\sim}{\rightarrow} Q_{I \cup\{n\}, s}(\mathbf{b})$, it will suffice to show that the nilpotent map $\bar{N}_{s}$ induced on $W=\Lambda_{\mathbf{r}} / \pi \Lambda_{\mathbf{r}}$ has Jordan block structure given by the partition of $n$.

The images $w_{i}$ of $\pi^{r_{i}} e_{i}$ form a basis for $W$, and the matrix for $\bar{N}_{s}$ with respect to this basis has the same form as does $N_{s}$. Thus, only the $(n, 1)$ and $(i, i+1)$ coefficients can possibly be nonzero. The image of $w_{i}$ under $\bar{N}_{s}$ is zero if and only if $r_{i}-r_{i-1}+\zeta_{i} \geq 1$, i.e. exactly when $c_{i}>0$. Suppose the $i$ th and $i^{\prime}$ th columns are nonzero with vanishing intervening columns (in increasing order with 1 following $n$ ). Then $c_{i}$ and $c_{i^{\prime}}$ are positive, and there is a cell containing precisely the walls $i$ through $i^{\prime}-1$. But a Jordan block of size $l$ is given by a string of $l-1$ consecutive nonzero columns surrounded by two zero columns, implying that Jordan blocks correspond to cells. The $b_{k}$ 's are the cell sizes, so the claim is proved.

Since $\mathcal{B}=\mathcal{F}_{[1, n-1]}$, we have $\chi\left(\mathcal{B}_{N}\right)=\left|Q_{[1, n], s}(\mathbf{b})\right|$. Each row of a $([1, n], s)$-intersection matrix sums to 1 , implying that the only nonzero entry in any row is a 1. Such a matrix is in the fiber over $\mathbf{b}$ if and only if there are exactly $b_{k} 1$ 's in the $k$ th column. Thus, an element of $Q_{[1, n], s}(\mathbf{b})$ is determined by arbitrarily selecting subsets of sizes $b_{1}, \ldots, b_{k}$ from the $n$ rows. This can be done in $\frac{n !}{b_{1} ! \cdots b_{s} !}=\frac{n !}{n_{1} ! \cdots n_{t} !}$ ways.

Remark. The smallest integer which can be used for $s$ in the above theorem for every nilpotent conjugacy class is $n+1$.

The bijection between the fiber $Y_{J s m}(\mathbf{r})$ and $Q_{J s}(\mathbf{b})$ extends to a one-to-one correspondence between the entire fixed point set and all orbits of $(J, s)$-intersection matrices.

Proposition 3.12. There is a bijection $Y_{J s m} \stackrel{\alpha}{\rightarrow} \tilde{Q}_{J s}$.

Proof. We define the function $\alpha$ on $Y_{J s m}(\mathbf{r})$ to be the composition of the maps $Y_{J s m}(\mathbf{r}) \rightarrow Q_{J s}^{\prime}(\mathbf{b}) \rightarrow Q_{J s}(\mathbf{b}) \rightarrow \tilde{Q}_{J s}$. The $s$-tuple $\mathbf{b} \in C_{n}^{s}$ is only defined up to cyclic permutation, but this ambiguity disappears upon passing to orbits.

Suppose $\alpha(\hat{\mathbf{r}})=\alpha\left(\hat{\mathbf{r}}^{\prime}\right)$. Note that $Q_{J s}(\mathbf{b})$ and $Q_{J s}\left(\mathbf{b}^{\prime}\right)$ have disjoint images in the orbit space unless $\mathbf{b}$ and $\mathbf{b}^{\prime}$ are cyclic permutations of each other. Hence, $\hat{\mathbf{r}}$ 
and $\hat{\mathbf{r}}^{\prime}$ must be in the same fiber $Q_{J s}(\mathbf{b})$. Propositions 3.8 and 3.10 now imply that $\hat{\mathbf{r}}=\hat{\mathbf{r}}^{\prime}$, so $\alpha$ is injective.

Given $Q=\left(q_{i k}\right) \in Q_{J s}$, define $\mathbf{b}$ by $b_{k}=\sum_{i=1}^{l} q_{i k}$. These are nonnegative integers, satisfying $\sum_{k=1}^{s} b_{k}=\sum_{k=1}^{s} \sum_{i=1}^{l} q_{i k}=\sum_{i=1}^{l} \sum_{k=1}^{s} q_{i k}=\sum_{i=1}^{l} p_{i}=n$. This shows that $\mathbf{b} \in C_{n}^{s}$ and $Q \in Q_{J s}(\mathbf{b})$. It is now clear that the corresponding orbit is in the image of $\alpha$, proving surjectivity.

We obtain the following theorem on Euler characteristics of fixed point varieties on affine flag manifolds:

Theorem 3.13. Assume that $\left(n\right.$, char $\left.^{\prime} k\right)=1$, and let $s$ be a positive integer relatively prime to $n$. Let $I=\left\{j_{1}<\cdots<j_{l}\right\} \subseteq[0, n-1]$ be a nonempty set of size $l$ with $p_{i}=j_{i+1}-j_{i}$ (where $\left.j_{l+1}=j_{1}+n\right)$. Then

$$
\chi\left(X_{I, N_{s}}\right)=s^{-1} \prod_{i=1}^{l}\left(\begin{array}{c}
s+p_{i}-1 \\
p_{i}
\end{array}\right) .
$$

In particular, $\chi\left(\hat{\mathcal{B}}_{N_{s}}\right)=s^{n-1}$ and if $\mathcal{K}$ is any variety of maximal parahoric subalgebras, $\chi\left(\hat{\mathcal{K}}_{N_{s}}\right)=\frac{(n+s-1) !}{n ! s !}$.

Proof. Let $J \subseteq[1, n]$ be the unique set such that $I=j_{1}+((J \backslash\{n\}) \cup\{0\})$ $(\bmod n)$. Note that $J=\left\{j_{2}-j_{1}, \ldots, j_{l}-j_{1}, n\right\}$, so the definition of the $p_{i}$ 's given in the statement coincides with the earlier definition. Since there is a bijection $Y_{J s j_{1}} \tilde{\rightarrow} \tilde{Q}_{J s}$ by proposition 3.12 , we have

$$
\chi\left(X_{I, N_{s}}\right)=\chi\left(\left(X_{I, N_{s}}\right)^{k^{*}}\right)=\left|Y_{J s j_{1}}\right|=\left|\tilde{Q}_{J s}\right|=\frac{\left|Q_{J s}\right|}{s} .
$$

The formula now follows from lemma 3.9

The varieties of maximal parahorics correspond to lattice chains of type $I$ with $I$ a singleton. In this case, $l=1$ and $p_{1}=n$, and the Euler characteristic is

$$
\frac{(n+s-1)}{n !(s-1) ! s}=\frac{(n+s-1) !}{n ! s !}
$$

We thus recover corollary 3.7 . The full affine flag variety $\hat{\mathcal{B}}$ is isomorphic to $X_{[0, n-1]}$. This gives $l=n$ and $p_{i}=1$ for each $i$, so $\chi\left(\hat{\mathcal{B}}_{N_{s}}\right)=s^{-1}\left(\begin{array}{l}s \\ 1\end{array}\right)^{n}=s^{n-1}$.

Remarks. 1. Let $\lambda_{1}, \ldots, \lambda_{n-1}$ be the roots of $\mathfrak{s l}_{n}(k)$ and $\lambda_{0}$ the affine root, and consider the subset of the positive Weyl chamber in an apartment of the affine building given by the conditions $\lambda_{i} \geq 0$ for $i \in[1, n-1]$ and $\lambda_{0} \leq s$. The Euler characteristic of $\hat{\mathcal{P}}_{N_{s}}$ is then just the number of simplices of type corresponding to the parahoric class in this region.

2. For $s=1$, the theorem implies that the Euler characteristic is 1 for each parahoric while if $n$ is odd and $s=2$, the Euler characteristics are given by

$$
\chi\left(X_{I, N_{b 2}}\right)=\frac{1}{2} \prod_{i=1}^{l}\left(p_{i}+1\right) .
$$

3. Lusztig and Smelt obtained the formulas for $\chi\left(\hat{\mathcal{B}}_{N_{1 s}}\right)$ and $\chi\left(\mathcal{L}_{0, N_{1 s}}\right)[\mathrm{LS}]$.

Finally, we recall a theorem due to Lusztig and Smelt: 
Theorem 3.14. The varieties $\hat{\mathcal{P}}_{N_{s}}$ have stratifications with finitely many strata, each of which is isomorphic to an affine space over $k$.

Proof. Since $N_{s}$ is conjugate to the nil-elliptic element $T_{s}$ considered by Lusztig and Smelt, their result carries over immediately [LS]. (Their argument, given for Iwahori and maximal parahoric subalgebras applies to the other parahoric subalgebras as well.)

Remark. The particular $k^{*}$-action considered by Lusztig and Smelt came from the $Q^{\prime}$-action $f(\lambda) \pi^{m} e_{i}=\lambda^{m n-i} \pi^{m} e_{i}$. The cells of the Bruhat decomposition for $\hat{\mathcal{P}}$ for this choice of ordered basis are just the cells of the Bialynicki-Birula plus decomposition for $f$. For example, consider the Bruhat cells of $\mathcal{L}_{m}$. Set $i, j \in[1, n]$ with $i \neq j$, set $\langle i, j\rangle=0$ if $i<j$ and 1 otherwise. The cells are indexed by $\mathbf{r} \in \mathbf{Z}^{n}$ such that $\sum_{i=1}^{n} r_{i}=m$, and they consist of those lattices with an $A$-basis $z_{1}, \ldots, z_{n}$ of the form $z_{j}=\pi^{r_{j}}\left(e_{j}+\sum_{i \neq j}\left(\sum_{l>0} a_{i j}^{l} \pi^{l+\langle i, j\rangle}\right) e_{i}\right)$, where $a_{i j}^{l} \in k$ for $i \neq j, l \geq 0$ and $a_{i j}^{l}=0$ if $l \geq r_{i}-r_{j}-\langle i, j\rangle$. It is easy to see that $f$ preserves the Bruhat cells and acts on the coordinates $a_{i j}^{l}$ for a given cell by sending them to $\lambda^{n l+n\langle i, j\rangle+j-i} a_{i j}^{l}$. The coefficient of $\lambda$ is always positive, so the limit as $\lambda$ goes to zero of $f(\lambda) L$ for any lattice in this cell is the diagonal lattice with $A$-basis $\pi^{r_{1}} e_{1}, \ldots, \pi^{r_{n}} e_{n}$. Since the cells of Lusztig and Smelt's stratification of $\hat{\mathcal{P}}_{T_{s}}$ are just the intersections of the fixed point variety with the Bruhat cells, we see that the stratification coincides with the plus decomposition.

Corollary 3.15. The étale cohomology of the varieties $\hat{\mathcal{P}}_{N_{s}}$ vanishes in odd degrees.

\section{4. $k^{*}$-ACTIONS ON FIXED POINT VARIETIES OF AFFINE FLAG MANIFOLDS: THE SYMPLECTIC ALGEBRAS}

We now consider the simple root systems $C_{n}$. Let $V$ be a $2 n$-dimensional vector space over $k$ endowed with a nondegenerate, alternating, bilinear form $\langle$,$\rangle . The$ symplectic group $S p(V)$ is the group of linear automorphisms of $V$ preserving the form. The corresponding Lie algebra is denoted $\mathfrak{s p}(V)$. A basis $\underline{e}=\left(e_{1}, \ldots, e_{2 n}\right)$ of $V$ is called symplectic if the subspaces $k e_{1} \oplus \cdots \oplus k e_{n}$ and $k e_{n+1} \oplus \cdots \oplus k e_{2 n}$ are isotropic, i.e. the restriction of the form to these subspaces is identically 0 , and $\left\langle e_{i}, e_{n+j}\right\rangle=\delta_{i j}$ for $i, j \in[1, n]$. It is well known that $V$ admits such a basis. Define a matrix

$$
J=\left(\begin{array}{cc}
0 & I_{n} \\
-I_{n} & 0
\end{array}\right) .
$$

A choice of symplectic basis identifies $S p(V)$ with $S p_{2 n}(k)=\left\{g \in G L_{2 n}(k) \mid g^{t} J g=\right.$ $J\}$, the semisimple, simply connected, algebraic group of type $C_{n}$. If $n=1$, this is just $S L_{2}(k)$. The symplectic Lie algebra with respect to this basis $\mathfrak{s p}_{2 n}(V)$ is the space of $2 n \times 2 n$ matrices $M$ satisfying $M^{t} J+J M=0$; equivalently,

$$
M=\left(\begin{array}{ll}
M_{11} & M_{12} \\
M_{21} & M_{22}
\end{array}\right)
$$

where $M_{22}=-M_{11}^{t}$ and the off-diagonal $n \times n$ blocks are symmetric.

The symplectic basis $\underline{e}$ determines a standard Borel subalgebra $\mathfrak{b}$ consisting of the matrices $M \in \mathfrak{s p}_{2 n}(k)$ such that $M_{21}=0$ and $M_{11}$ is upper triangular, using the notation of (4-1). (The subalgebra of upper triangular matrices in $\mathfrak{s p}_{2 n}(k)$ is 
not a Borel subalgebra.) Let $\hat{\mathfrak{b}} \subset \mathfrak{s p}_{2 n}(A)$ be the corresponding standard Iwahori subalgebra. We define the algebras $\hat{\mathfrak{q}}_{i} \subset \mathfrak{s p}_{2 n}(F)$ for $i \in[0, n]$ by

$$
\hat{\mathfrak{q}}_{i}=\mathfrak{s p}_{2 n}(F) \cap\left(\begin{array}{cccc}
i & n-i & i & n-i \\
A & A & \pi^{-1} A & A \\
\pi A & A & A & A \\
\pi A & \pi A & A & \pi A \\
\pi A & A & A & A
\end{array}\right){ }_{n-i} i
$$

These are the maximal parahoric subalgebras $\hat{\mathfrak{q}}_{i}$ containing our fixed Iwahori subalgebra. Their stabilizers $\hat{Q}_{i} \subseteq S p_{2 n}(F)$ have the same block form. The parahoric subalgebras containing $\hat{\mathfrak{b}}$ are now given by $\hat{\mathfrak{p}}_{I}=\bigcap_{i \notin I} \hat{\mathfrak{q}}_{i}$ with stabilizer $\hat{P}_{I}=\bigcap_{i \notin I} \hat{Q}_{i}$ for each proper subset $I$ of $[0, n]$; this notation is compatible with notation of section 2 for a suitable choice of simple reflections for $\hat{W}$, indexed by $[0, n]$. The affine flag manifolds $\hat{\mathcal{P}}_{I}$ for $S p\left(V_{F}\right)$ are identified with the quotient spaces $S p\left(V_{F}\right) / \hat{P}_{I}$.

We will again interpret the spaces of parahoric subalgebras as chains of $A$-lattices in $V_{F}$. Let $L \subset V_{F}$ be a lattice. The set $L^{*}=\left\{v \in V_{F} \mid\langle v, L\rangle \subseteq A\right\}$ is called the dual lattice to $L$; it is a lattice with valuation $-v(L)$.

Definition. A lattice $L$ is called symplectic if the set of lattices generated from $L$ by homothety and duality (or equivalently, the set of lattices homothetic to $L$ or $\left.L^{*}\right)$ is totally ordered by inclusion, i.e. a chain. The variety of symplectic lattices is denoted $\mathcal{L}^{S p}$.

The symplectic group $S p\left(V_{F}\right)$ acts on $\mathcal{L}^{S p}$, and the orbits of this action are the subvarieties $\mathcal{L}_{m}^{S p} \stackrel{\text { def }}{=} \mathcal{L}^{S p} \cap \mathcal{L}_{m}$. We can apply the theory of alternating forms on free modules over a principal ideal domain (see theorem IX.5.1 in [B1]) to obtain a more explicit characterization of these orbits. For $m \geq 0$, write $m=q n+r$ with $r \in[0, n-1]$. The variety $\mathcal{L}_{m}^{S p}$ is the set of lattices contained in their duals and admitting a basis $\underline{z}$ such that $\bigoplus_{i=1}^{n} A z_{i}$ and $\bigoplus_{i=n+1}^{2 n} A z_{i}$ are isotropic submodules and $\left\langle z_{i}, z_{n+j}\right\rangle$ equals $\delta_{i j} \pi^{q}$ for $i \in[1, n-r]$ and $\delta_{i j} \pi^{q+1}$ for $i>n-r$. For $m \leq 0$, $\mathcal{L}_{m}^{S p}$ is the set of lattices whose duals are in $\mathcal{L}_{|m|}^{S p}$. In particular, $\mathcal{L}_{0}^{S p}$ is the space of self-dual lattices.

As in the case of $S L\left(V_{F}\right)$, if $m \equiv m^{\prime}(\bmod 2 n)$, then $\mathcal{L}_{m}^{S p} \cong \mathcal{L}_{m^{\prime}}^{S p}$ as an $S p\left(V_{F}\right)$ variety. In addition, the correspondence $L \mapsto L^{*}$ defines an $S p\left(V_{F}\right)$-isomorphism $\mathcal{L}_{m}^{S p} \rightarrow \mathcal{L}_{-m}^{S p}$. Thus, by identifying a symplectic lattice $L$ with the totally ordered set $\left\{\pi^{q} L, \pi^{q^{\prime}} L^{*} \mid q, q^{\prime} \in \mathbf{Z}\right\}$, we can view $\mathcal{L}_{m}^{S p}$ as a variety of chains of symplectic lattices. More generally, we make the following definition:

Definition. Let $I$ be a nonempty subset of $[0, n]$. The space of (partial) symplectic lattice chains of type $I$ is the set

$$
\begin{aligned}
X_{I}^{S p}=\left\{\left(L_{i}\right) \mid i \in I \cup-I\right. & (\bmod 2 n), L_{i} \in \mathcal{L}_{i}^{S p}, \\
& \left.L_{i} \supset L_{j} \Longleftrightarrow i<j, L_{i+2 n}=\pi L_{i}, \text { and } L_{-i}=L_{i}^{*}\right\} .
\end{aligned}
$$

The $S p\left(V_{F}\right)$-action on the $\mathcal{L}_{m}^{S p}$ 's extends to an action on the $X_{I}^{S p}$ 's. Note that $X_{I}^{S p}$ is a subvariety of $X_{I \cup(2 n-I) \backslash\{2 n\}}$, the set of lattice chains of type $I \cup(2 n-I) \backslash\{2 n\}$.

An argument similar to that sketched in section 3 shows that the varieties $X_{I}^{S p}$ are the affine flag manifolds for $S p\left(V_{F}\right)$ (see proposition 4.6 and theorem 4.8 in $[\underline{\underline{S}}$ ): 
Theorem 4.1. For each proper subset of $[0, n], \hat{\mathcal{P}}_{I} \cong X_{I^{c}}^{S p}$ as an $S p\left(V_{F}\right)$-variety. Moreover, if $0 \in I$, then the fiber over the self-dual lattice $L$ of the projection $X_{I}^{S p} \rightarrow X_{\{0\}}^{S p}=\mathcal{L}_{0}^{S p}$ is isomorphic as an $S p(L / \pi L)$-variety to $\mathcal{F}_{I \backslash\{0\}}^{S p}(L / \pi L)$, the variety of isotropic flags of type $I \backslash\{0\}$ in $L / \pi L$.

Let $\tilde{Q}_{S p}^{\prime}=G S p(V) \times k^{*}$, where $G S p(V)$ is the group of symplectic similitudes of $V$. We call a $Q^{\prime}$-action $f$ a $\tilde{Q}_{S p}^{\prime}$-action if $f\left(k^{*}\right) \subseteq \tilde{Q}_{S p}^{\prime}$; these are precisely the $Q^{\prime}$-actions which preserve the space of symplectic lattices. Such a homomorphism induces a $Q_{S p}^{\prime}$-action via the projection $G S p(V) \rightarrow P G S p(V)$ as described in section [2]

We now assume that char $k \neq 2$. If $N \in \mathfrak{s p}\left(V_{F}\right)$ is semisimple, its eigenvalues must be of the form $\pm a_{1}, \ldots, \pm a_{n}$ where $a_{i} \in \bar{F}$. The characteristic polynomial $\operatorname{char}_{N}(\mu)=\prod_{i=1}^{n}\left(\mu^{2}-a_{i}^{2}\right)$ is an element of $F\left[\mu^{2}\right]$, and we define a polynomial $h_{N} \in F[\mu]$ by $h\left(\mu^{2}\right)=\operatorname{char}_{N}(\mu)$. The eigenvalues of ad $N: \mathfrak{g} \rightarrow \mathfrak{g}$ are $\pm a_{i} \pm a_{j}$ for $i<j, \pm 2 a_{i}$, and 0 with multiplicity $n$. The endomorphism $N$ is regular semisimple if the kernel of $N$ has dimension $n$; this occurs when the $a_{i}^{2}$ 's are distinct and nonzero. It is well-known that the characteristic polynomial, or equivalently $h_{N}$, determines the $S p\left(V_{\bar{F}}\right)$-conjugacy classes of regular semisimple elements of $\mathfrak{s p}\left(V_{\bar{F}}\right)$. Proposition 3.2 shows that the same is true for regular semisimple classes in $\mathfrak{s p}\left(V_{F}\right)$.

Let $\mathcal{C}$ be a regular semisimple conjugacy class in $\mathfrak{s p}\left(V_{F}\right)$, and suppose that there exists $N \in \mathcal{C}$ and a $Q_{S p}^{\prime}$-action $f$ almost commuting with $N$. The endomorphism $N$ is also regular semisimple viewed as an element of $\mathfrak{g l}\left(V_{F}\right)$, so by theorem 2.4, $\operatorname{char}_{N}$ is a homogeneous polynomial in $\mu$ and $\pi^{q^{\prime}}$ for some nonzero $q^{\prime} \in \frac{1}{2 n-1} \mathbf{Z} \cup \frac{1}{2 n} \mathbf{Z}$. Moreover, since $\operatorname{det} N \neq 0$, the $\mu^{0} \pi^{2 n q^{\prime}}$ term does not vanish, implying that $2 n q^{\prime} \in$ Z. It is immediate that $h_{N}$ is homogeneous in $\mu$ and $\pi^{q}$ where $q=2 q^{\prime} \in \frac{1}{n} \mathbf{Z}$. The converse can also be proven by an argument similar to the proof of theorem 2.4

Theorem 4.2. Let $\mathcal{C}$ be a regular semisimple conjugacy class in $\mathfrak{s p}\left(V_{F}\right)$. Then there is a representative $N \in \mathcal{C}$ almost commuting with a $Q_{S p}^{\prime}$-action if and only if $h_{\mathfrak{C}}(\mu)$ is a homogeneous polynomial in $\mu$ and $\pi^{q}$ for some nonzero $q \in \frac{1}{n} \mathbf{Z}$. $\mathcal{C}$ is nil if and only if $q>0$.

If we write $h_{\mathcal{C}}(\mu)=\sum_{i=0}^{n} c_{i} \pi^{q i} \mu^{n-i}$ and identify $\mathfrak{s p}\left(V_{F}\right)$ with $\mathfrak{s p}_{2 n}(F)$ by choosing a symplectic basis for $V$, we can define a representative $N=\left(a_{i j}\right)$ for $\mathcal{C}$ by:

$$
a_{i j}= \begin{cases}1 & \text { if } i=j+1, j \in[1, n-1] \text { or }(i, j)=(1, n+1), \\ -1 & \text { if } j=i+1, i \in[n+1,2 n-1], \\ (-1)^{i} c_{i} \pi^{q i} & \text { if } i=j+n, j \in[1, n], \\ 0 & \text { otherwise. }\end{cases}
$$

Corollary 4.3. The regular semisimple conjugacy classes in $\mathfrak{s p}\left(V_{F}\right)$ obtained from almost commuting pairs $(N, f)$ with $f$ a $Q_{S p}^{\prime}$-action are precisely those with $h_{\mathfrak{C}}(\mu)=$ $\prod_{i=1}^{r}\left(\mu^{d}-b_{i} \pi^{s}\right)$ where $r d=n,(s, d)=1,\left(\right.$ char $\left.^{\prime} k, d\right)=1$, and the $b_{i}$ 's are distinct elements of $k^{*}$.

Proof. If $\mathcal{C}$ is such a class, then $h_{\mathcal{C}}$ is a homogeneous polynomial in $\mu$ and $\pi^{q}$. Let $q=s / d$ with $(s, d)=1$ and $d>0$. All the eigenvalues are nonzero, so by corollary [2.3, each is homogeneous of degree $q$. If $a \pi^{q}$ is an eigenvalue, then it satisfies the irreducible polynomial $\mu^{d}-a^{d} \pi^{s}$. Consequently, every irreducible factor of $h_{\mathcal{C}}$ has degree $d$, implying that $n=r d$ for some positive integer $r$. Since 
these $r$ factors must be distinct, we obtain the $r$ different $b_{i}$ 's. Finally, the factors are separable polynomials if and only if $\left(\operatorname{char}^{\prime} k, d\right)=1$.

We now find an explicit criterion for ellipticity of regular semisimple conjugacy classes in $\mathfrak{s p}\left(V_{F}\right)$. Write $h_{N}=h_{1} \ldots h_{l}$ where the $h_{i}$ 's are distinct irreducible polynomials. If we set $h_{i}^{\prime}(\mu)=h_{i}\left(\mu^{2}\right)$, then $\operatorname{char}_{N}=h_{1}^{\prime} \ldots h_{l}^{\prime}$. Let $E_{i}=F[\mu] /\left(h_{i}\right)$; it is a field extension of degree $n_{i}=\operatorname{deg} h_{i}$ over $F$. By the Chinese remainder theorem, $E=F[\mu] /\left(h_{N}\right) \cong \bigoplus_{i=1}^{l} E_{i}$. Similarly, if we set $E_{i}^{\prime}=F[\mu] /\left(h_{i}^{\prime}\right), E^{\prime}=$ $F[\mu] /\left(\operatorname{char}_{N}\right) \cong \bigoplus_{i=1}^{l} E_{i}^{\prime}$. The $F$-algebra injection $E \hookrightarrow E^{\prime}$ given by $\mu \mapsto \mu^{2}$ induces injections $E_{i} \hookrightarrow E_{i}^{\prime}$ for each $i$, making $E_{i}^{\prime}$ into a 2-dimensional $E_{i}$-algebra. If $h_{i}^{\prime}$ is irreducible, then $E_{i}^{\prime}$ is a field. Otherwise, let $g_{1}, \ldots, g_{t}$ be the monic, irreducible factors of $h_{i}^{\prime}$. If $g_{j}(\mu) \mid h_{i}^{\prime}$, then so does $g_{j}(-\mu)$. Moreover, if the ideals generated by $g_{j}(\mu)$ and $g_{j}(-\mu)$ were equal, then $g_{j}$ would be a polynomial in $\mu^{2}$ and would give rise to a proper irreducible factor of $h_{i}$. We can thus assume that $g_{2}(\mu)=(-1)^{n} g_{1}(-\mu)$; the coefficient guarantees that $g_{1}$ and $g_{2}$ are simultaneously monic. But now $g_{1} g_{2}$ is a polynomial in $\mu^{2}$, and this contradicts irreducibility of $h_{i}$ unless $h_{i}^{\prime}=g_{1} g_{2}$. Since $g_{1}$ and $g_{2}$ are distinct, $E_{i}^{\prime} \cong F[\mu] /\left(g_{1}\right) \oplus F[\mu] /\left(g_{2}\right) \cong E_{i} \oplus E_{i}$. The last isomorphism follows because the homomorphisms $E_{i} \rightarrow F[\mu] /\left(g_{j}\right)$ for $j \in[1,2]$ are nonzero maps between fields which have the same dimension as $F$ vector spaces.

Proposition 4.4. Let $N$ be a regular semisimple element of $\mathfrak{s p}\left(V_{F}\right)$. Then $N$ is elliptic if and only if $F[\mu] /\left(h_{N}\right)$ and $F[\mu] /\left(\right.$ char $\left._{N}\right)$ decompose into direct sums of the same number of fields. Equivalently, for each irreducible factor $h_{i}$ of $h_{N}$, $h_{i}^{\prime}(\mu)=h_{i}\left(\mu^{2}\right)$ is also irreducible.

Proof. We construct an element $N_{i} \in \mathfrak{s p}_{2 n_{i}}(F)$ with $h_{N_{i}}=h_{i}$ by the procedure of theorem 4.2. Using the injection $\prod_{i=1}^{l} \mathfrak{s p}_{2 n_{i}}(F) \hookrightarrow \mathfrak{s p}_{2 n}(F)$, we obtain a 'blockdiagonal' matrix $N^{\prime} \in \mathfrak{s p}_{2 n}(F)$ with $h_{N^{\prime}}=h_{N}$. (The restriction of $N^{\prime}$ to $W_{1}=$ $\bigoplus_{j=1}^{n_{1}}\left(F e_{j} \oplus F e_{n+j}\right)$ is $N_{1}$ and so on.) Since $N$ is regular semisimple, $N$ and $N^{\prime}$ are conjugate, and there is a symplectic basis $\underline{w}$ of $V_{F}$ in which $N$ is given by $N^{\prime}$.

Suppose some $h_{i}^{\prime}$ is reducible. Since $V_{F}=\bar{W}_{1} \perp \cdots \perp W_{l}$, it suffices to exhibit an $F$-cocharacter $F^{*} \rightarrow Z_{S p\left(W_{i}\right)}\left(N_{i}\right)$. We thus assume without loss of generality that $h_{N}$ is irreducible, but char $N$ is not. We then have $\operatorname{char}_{N}=g_{1} g_{2}$ with $g_{2}(\mu)=$ $(-1)^{n} g_{1}(-\mu)$. Construct a matrix $M \in \mathfrak{g l}_{n}(F)$ with characteristic polynomial $g_{1}$ as in the proof of theorem [2.4. It has $n$ distinct eigenvalues $a_{1}, \ldots, a_{n} \in \bar{F}$ and $g_{1}(\mu)=\prod_{i=1}^{n}\left(\mu-a_{i}\right)$. The transpose $M^{t}$ has the same characteristic polynomial and eigenvalues, so $\operatorname{char}_{-M^{t}}(\mu)=\prod_{i=1}^{n}\left(\mu+a_{i}\right)=(-1)^{n} g_{1}(-\mu)=g_{2}(\mu)$. The block-diagonal matrix $M^{\prime}=\operatorname{diag}\left(M,-M^{t}\right) \in \mathfrak{s p}_{2 n}(F)$ has the same characteristic polynomial as $N$, and again invoking regularity, $N$ and $M^{\prime}$ are $S p_{2 n}(F)$-conjugate. Since the $F$-cocharacter $F^{*} \rightarrow \mathbf{Z}_{S p_{2 n}(F)}\left(M^{\prime}\right)$ given by $x \mapsto \operatorname{diag}\left(x I_{n}, x^{-1} I_{n}\right)$ is nontrivial, $N$ is not elliptic.

Conversely, suppose $N$ is not elliptic, so that there exists a nontrivial cocharacter $F^{*} \stackrel{\phi}{\rightarrow} Z_{S p_{2 n}(F)}(N)$. Using the above orthogonal decomposition of $V_{F}$, we obtain cocharacters $F^{*} \stackrel{\phi_{i}}{\longrightarrow} Z_{S p\left(W_{i}\right)}\left(N_{i}\right)$. Since at least one $\phi_{i}$ is nontrivial, we can assume without loss of generality that $h_{N}$ is irreducible. If $\operatorname{char}_{N}$ is also irreducible, then there cannot even be a nontrivial cocharacter $F^{*} \rightarrow Z_{S L_{2 n}(F)}(N)$. Thus, $\operatorname{char}_{N}$ is reducible as desired. 
Suppose that $N$ is nil-elliptic and almost commutes with a $Q_{S p^{-a c t i o n}}^{\prime}$ The irreducible factors of $h_{N}$ are all of the form $h_{i}(\mu)=\mu^{d}-b_{i} \pi^{s}$ with $b_{i} \in F^{*}$, $(d, s)=1$, and $s>0$. The polynomial $h_{i}^{\prime}(\mu)=\mu^{2 d}-b_{i} \pi^{s}$ is irreducible if and only if $(2 d, s)=1$, i.e. when $(d, s)=1$ and $s$ is odd. Applying the previous result, we have:

Proposition 4.5. The nil-elliptic conjugacy classes in $\mathfrak{s p}\left(V_{F}\right)$ obtained from almost commuting pairs $(N, f)$ with $f$ a $Q_{S p}^{\prime}$-action are precisely those with $h_{\mathrm{C}}(\mu)=$ $\prod_{i=1}^{r}\left(\mu^{d}-b_{i} \pi^{s}\right)$ where $s>0, r d=n,(s, 2 d)=1,\left(\right.$ char' $\left.^{\prime}, d\right)=1$, and the $b_{i}$ 's are distinct elements of $k^{*}$. If $N$ is the standard matrix representative of theorem 4.2. $f$ almost commutes with exponent $s$ with the diagonal $Q_{S p}^{\prime}$-action $f$ given by $\nu(m, i)=2 d m+i s$ and $\nu(m, n+i)=2 d m+(1-i) s$ for $i \in[1, n]$.

The varieties $\left(\hat{\mathcal{P}}_{N}\right)^{k^{*}}$ are in general much simpler than the $\hat{\mathcal{P}}_{N}$ 's, but, because the $k^{*}$-actions of the above proposition do not always have discrete fixed point sets, they are not necessarily finite. It is possible to identify the fixed lattices precisely; the off-diagonal entries which may be nonzero as well as the terms present in these Laurent series are fully determined by $r$.

We now restrict ourselves to the case $r=1, d=n$, and $\left(\operatorname{char}^{\prime} k, 2 n\right)=(s, 2 n)=$ 1. We let $N_{b s}$ denote the standard representative of the class with $h_{\mathcal{C}}(\mu)=\mu^{n}-b \pi^{s}$ given in theorem 4.2. (This does not seriously conflict with our previous notation in section 3 because the element of $\mathfrak{s l}_{2 n}(F)$ given by the two definitions are in the same $S L_{2 n}(F)$-conjugacy class. Also, note that the fixed point varieties for $N_{b s}$ viewed as an element of $\mathfrak{s p}_{2 n}(F)$ are closed subvarieties of the analogous fixed point varieties with $N_{b s}$ viewed as an element of $\mathfrak{s l}_{2 n}(F)$; in particular, they are algebraic varieties even in positive characteristic.) Again, we can assume $b=1$, and we set $N_{s} \stackrel{\text { def }}{=} N_{1 s}$. By the remark after lemma 3.4, the $Q_{S p}^{\prime}$-action $f$ fixes only the diagonal lattices. The set of symplectic lattice chains of type $J$ composed of diagonal lattices will be denoted by $\mathcal{D}_{J}^{S p}$; in particular, we set $\mathcal{D}_{m}^{S p}=\mathcal{D}_{m} \cap \mathcal{L}_{m}^{S p}$. Given $I \subseteq[1, n]$, let $\delta_{i}^{I}=1$ if $i \in I$ and $\delta_{i}^{I}=0$ otherwise. It is immediate that for $0 \leq m \leq n, \Lambda_{\mathbf{r}} \in \mathcal{D}_{m}^{S p}$ if and only if there exists $I \subseteq[1, n]$ such that $|I|=m$ and $r_{n+i}=-r_{i}+\delta_{i}^{I}$.

We will again apply the theorem of Bialynicki-Birula to give explicit combinatorial interpretations for the Euler characteristics $\chi\left(\hat{\mathcal{P}}_{N_{s}}\right)$. Because of the considerable loss of symmetry involved in the passage to the symplectic algebras, the resulting counting problems are more complicated than in the special linear case.

We begin by determining the fixed point sets $\left(\mathcal{L}_{m, N_{s}}^{S p}\right)^{k^{*}}$. Suppose that $\Lambda=$ $\Lambda_{\mathbf{r}} \in \mathcal{D}_{m}^{S p}$ is fixed by $N_{s}$. We must have $\pi^{r_{i}} e_{i+1} \in A \pi^{r_{i+1}} e_{i+1}$ and $-\pi^{r_{n+i+1}} e_{n+i} \in$ $A \pi^{r_{n+i}} e_{n+i}$ for $i \in[1, n-1], \pi^{r_{n+1}} e_{1} \in A \pi^{r_{1}} e_{1}$, and $(-1)^{n+1} \pi^{r_{n}+s} e_{2 n} \in A \pi^{r_{2 n}} e_{2 n}$. As a result, $\left(\mathcal{L}_{m, N_{s}}^{S p}\right)^{k^{*}}=\left\{\Lambda_{\mathbf{r}} \mid \mathbf{r} \in R_{s m}^{S p}\right\}$ where $R_{s m}^{S p}$ is the union over $I \subseteq[1, n]$ of size $m$ of the disjoint sets

$$
\begin{array}{r}
R_{s I}^{S p}=\left\{\mathbf{r} \in \mathbf{Z}^{2 n} \mid r_{n} \leq r_{n-1} \leq \cdots \leq r_{1} \leq r_{n+1} \leq r_{n+2} \leq \cdots \leq r_{2 n} \leq r_{n}+s\right. \\
\text { and } \left.r_{n+i}=-r_{i}+\delta_{i}^{I}\right\} .
\end{array}
$$

Let $R_{s m}$ be the set of $2 n$-tuples such that $\left(\mathcal{L}_{m, N_{s}}\right)^{k^{*}}=\left\{\Lambda_{\mathbf{r}} \mid \mathbf{r} \in R_{s m}\right\}$. This is the same definition as in (3-4) up to the numbering of the entries. Thus, we have a bijection $R_{s m} \stackrel{\phi}{\rightarrow} C_{s m}=C_{s m}^{2 n}$ as in lemma 3.6. The indexing $\mathbf{c}=\left(c_{1}, \ldots, c_{2 n}\right)$ is not convenient here, and we will write instead

$$
\left(c_{1}, \ldots, c_{2 n}\right)=\left(q_{n}, q_{n-1}, \ldots, q_{1}, q_{0}, q_{-1}, \ldots, q_{-(n-1)}\right) .
$$


The function $\phi$ is given by $q_{0}=r_{n+1}-r_{1}, q_{n}=r_{n}-r_{2 n}+s, q_{i}=r_{i}-r_{i+1}$, and $q_{-i}=r_{n+i+1}-r_{n+i}$ for $i \in[1, n-1]$.

We now determine $\phi\left(R_{s m}^{S p}\right)$. Given a set $I \subseteq[1, n]$ and $i \in[1, n-1]$, let $\epsilon_{i}^{I}=$ $\delta_{i+1}^{I}-\delta_{i}^{I}$. Define

$$
\begin{aligned}
C_{s I}^{S p}=\left\{\mathbf{c} \in C_{s}^{2 n} \mid q_{-i}=q_{i}+\epsilon_{i}^{I} \text { for } i \in[1, n-1], q_{n} \text { is even } \Longleftrightarrow n \in I,\right. \\
\text { and } \left.q_{0} \text { is odd } \Longleftrightarrow 1 \in I\right\}
\end{aligned}
$$

and

$$
C_{s m}^{S p}=\bigcup_{\substack{I \subseteq[1, n] \\|\bar{I}|=m}} C_{s I}^{S p}
$$

The union is clearly disjoint. Note that it is not a priori obvious that $C_{s m}^{S p} \subseteq C_{s m}$.

We will show that $\phi\left(R_{s m}^{S p}\right)=C_{s m}^{S p}$. We need the following lemmas:

Lemma 4.6. For any $n \geq 1$ and any $I \subseteq[1, n]$ with $|I|=m$,

$$
\sum_{i=1}^{n-1} i \epsilon_{i}^{I}= \begin{cases}n-m & \text { if } n \in I, \\ -m & \text { if } n \notin I .\end{cases}
$$

Proof. We have

$$
\sum_{i=1}^{n-1} i \epsilon_{i}^{I}=\sum_{i=1}^{n-1} i\left(\delta_{i+1}^{I}-\delta_{i}^{I}\right)=n \delta_{n}^{I}-\sum_{i=1}^{n} \delta_{i}^{I}=n \delta_{n}^{I}-m
$$

as desired.

Lemma 4.7. $C_{s m}^{S p} \subseteq C_{s m}$.

Proof. We must show that $\sum_{i=1}^{2 n}(l+i) c_{i} \equiv-m+(l+1) s(\bmod 2 n)$ for some $l \in \mathbf{Z}$. Set $l=-(n+1)$. The sum is then $-n q_{n}+0 q_{0}+\sum_{i=1}^{n-1} i\left(q_{-i}-q_{i}\right)=-n q_{n}+\sum_{i=1}^{n-1} i \epsilon_{i}^{I}$. By lemma 4.6, this is congruent to $n-m$ regardless of the parity of $q_{n}$. Since $s$ is odd, $-m-n s \equiv-m+n(\bmod 2 n)$, and the result follows.

We are now ready to prove:

Proposition 4.8. The map $\phi$ induces bijections $R_{s I}^{S p} \sim C_{s I}^{S p}$ for each $I \subseteq[1, n]$ and hence bijections $R_{s m}^{S p} \sim C_{s m}^{S p}$ for all $m \in[0, n]$.

Proof. It is clear from lemma 4.7 that $\phi\left(R_{s I}^{S p}\right) \subseteq C_{s I}^{S p} \subseteq C_{s|I|}^{S p}$, so it will suffice to show that $\phi^{-1}\left(C_{s I}^{S p}\right) \subseteq R_{s I}^{S p}$. Choose $\mathbf{c} \in C_{s I}^{S p}$, and let $\mathbf{r}=\phi^{-1}(\mathbf{c})$. We will show by downward induction on $i$ that $r_{i}+r_{n+i}=\delta_{i}^{I}$.

Using the formula for $\phi^{-1}$ given in lemma 3.6, we have

$$
\begin{aligned}
r_{2 n} & =\frac{m-s+\sum_{j=-n+1}^{n}(n+1-j) q_{j}}{2 n} \text { and } \\
r_{n} & =\frac{m-s+q_{n}+\sum_{j=-n+1}^{n-1}(-n+1-j) q_{j}}{2 n} .
\end{aligned}
$$


Adding, we get

$$
\begin{aligned}
r_{n}+r_{2 n} & =\frac{m-s+q_{n}+\sum_{j=-n+1}^{n-1}(1-j) q_{j}}{n} \\
& =\frac{m-s+q_{n}+\sum_{j=-n+1}^{n-1} q_{j}-\sum_{j=-n+1}^{n-1} j q_{j}}{n} \\
& =\frac{m-s+q_{n}+\left(s-q_{n}\right)+\sum_{j=1}^{n-1} j \epsilon_{j}^{I}}{n}=\delta_{n}^{I},
\end{aligned}
$$

where the last step is lemma 4.6.

Now suppose $r_{i+1}+r_{n+i+1}=\delta_{i+1}^{I}$. By the formula for $\phi^{-1}, r_{i}+r_{n+i}=r_{i+1}+$ $r_{n+i+1}+\left(q_{i}-q_{-i}\right)$. If $i+1 \in I$, then the term in parentheses is -1 (resp. 0 ) for $i \notin I$ (resp. $i \in I)$ as desired. The case $i+1 \notin I$ is checked similarly.

Given a set $I \subseteq[1, n]$, define $\hat{I}=I \cup\{n+1\}$. Let

$$
G_{s I}^{S p}=\left\{\mathbf{p}=\left(p_{0}, \ldots, p_{n}\right) \in C_{s}^{n+1} \mid p_{i} \text { is even } \Longleftrightarrow i, i+1 \in \hat{I} \text { or } i, i+1 \notin \hat{I}\right\},
$$

and let $G_{s m}^{S p}$ denote the (disjoint) union of these sets over all $I$ of size $m$. Define a function $C_{s I}^{S p} \stackrel{\psi}{\rightarrow} G_{s I}^{S p}$ by $\mathbf{q} \mapsto\left(q_{0}, q_{1}+q_{-1}, \ldots, q_{n-1}+q_{-(n-1)}, q_{n}\right)$. We also have a $\operatorname{map} G_{s I}^{S p} \rightarrow C_{s I}^{S p}$ with $q_{0}=p_{0}, q_{n}=p_{n}$, and for $i \in[1, n-1]$,

$$
\left(q_{i}, q_{-i}\right)= \begin{cases}\left(\left[p_{i} / 2\right],\left[p_{i} / 2\right]+1\right) & \text { if } i \notin I, i+1 \in I, \\ \left(\left[p_{i} / 2\right]+1,\left[p_{i} / 2\right]\right) & \text { if } i \in I, i+1 \notin I, \\ \left(\left[p_{i} / 2\right],\left[p_{i} / 2\right]\right) & \text { otherwise. }\end{cases}
$$

It is easy to see that these maps are inverses, so we have proven

Lemma 4.9. The function $\psi$ defines bijections $C_{s I}^{S p} \sim G_{s I}^{S p}$ and $C_{s m}^{S p} \sim G_{s m}^{S p}$ for all $I \subseteq[1, n]$ and $m \in[0, n]$.

For any $I \subseteq[1, n]$ with $|I|=m$, we can interpret $G_{s I}^{S p}$ as the set of distinct arrangement of $s$ balls in $n+1$ boxes with the parity of the number of balls in each box determined by $I$. Suppose $l$ of the boxes contain an odd number of balls. Since these boxes are nonempty and $s=2 t+1$ is odd, $l=2 a+1$ for some $a \in[0, t]$. Note that $\sum_{i=0}^{n}\left[p_{i} / 2\right]=(s-l) / 2=t-a$, and any such arrangement is uniquely determined by the $\left[p_{i} / 2\right]$ 's. Thus, there is a bijection from $G_{s I}^{S p}$ to $C_{t-a}^{n+1}$, implying that $\left|G_{s I}^{S p}\right|=\left(\begin{array}{c}n+t-a \\ n\end{array}\right)$.

Let $h_{n, m, a}$ be the number of subsets $I \subseteq[1, n]$ with $|I|=m$ such that $2 a+1$ of the $p_{i}$ 's are odd. A succession in $\hat{I}$ is a pair of numbers $i, i+1 \in \hat{I}$. Let $g_{n, m, j}$ be the number of $I$ 's of size $m$ such that $\hat{I}$ has $j$ successions. Write $\hat{I}=\left\{i_{1}<\right.$ $\left.\cdots<i_{m}<i_{m+1}=n+1\right\}$, and partition it into maximal sequences of consecutive integers $\left\{i_{l_{j-1}+1}<\cdots<i_{l_{j}}\right\}$. Suppose there are $d$ sequences in the partition. Since $0 \notin \hat{I}$ and $n+1 \in \hat{I}, l_{0}=0$ and $l_{d}=m+1$. Each maximal sequence contributes $l_{j}-l_{j-1}-1$ successions to $\hat{I}$, so there are a total of $l_{d}-l_{0}-d=m+1-d$ successions. The boxes containing an odd number of balls are those indexed by $i_{l_{j-1}+1}-1$ for $j \in[1, d]$ and $i_{l_{j}}$ for $j \in[1, d-1]$. Hence, there are $2(d-1)+1$ such boxes, i.e. $a=d-1$, implying that $h_{n, m, a}=g_{n, m, m-a}$. We must have $0 \leq a \leq m$, since the number of successions is evidently between 0 and $m$. 
Lemma 4.10. For each $j \in[0, m]$,

$$
g_{n, m, j}=\left(\begin{array}{c}
m \\
j
\end{array}\right)\left(\begin{array}{c}
n-m \\
m-j
\end{array}\right) \text {. }
$$

Proof. Let $f_{n, m, j}$ be the number of size $m$ subsets $I \subseteq[1, n]$ having $j$ successions. It is well known (see $[\mathbb{R}$, p. 12]) that

$$
f_{n, m, j}=\left(\begin{array}{c}
m-1 \\
j
\end{array}\right)\left(\begin{array}{c}
n-m+1 \\
m-j
\end{array}\right) .
$$

We see that $g_{n, m, j}$ is the number of subsets of $[1, n+1]$ of size $m+1$ which contain the element $n+1$ and have $j$ successions. This collection of subsets consists of those size $m+1$ subsets with $j$ successions which are not already subsets of $[1, n]$. Thus,

$$
\begin{aligned}
g_{n, m, j}=f_{n+1, m+1, j}-f_{n, m+1, j} & =\left(\begin{array}{c}
m \\
j
\end{array}\right)\left(\begin{array}{c}
n-m+1 \\
m+1-j
\end{array}\right)-\left(\begin{array}{c}
m \\
j
\end{array}\right)\left(\begin{array}{c}
n-m \\
m+1-j
\end{array}\right) \\
& =\left(\begin{array}{c}
m \\
j
\end{array}\right)\left(\begin{array}{c}
n-m \\
m-j
\end{array}\right) . \square
\end{aligned}
$$

We can finally compute $\left|G_{s m}^{S p}\right|$.

\section{Proposition 4.11.}

$$
\left|G_{s m}^{S p}\right|=\left(\begin{array}{c}
{[s / 2]+m} \\
m
\end{array}\right)\left(\begin{array}{c}
{[s / 2]+n-m} \\
n-m
\end{array}\right)
$$

Proof. We have

$$
\begin{aligned}
\left|G_{s m}^{S p}\right|=\sum_{\substack{I \subseteq[1, n] \\
|\bar{I}|=m}}\left|G_{s I}^{S p}\right| & =\sum_{a=0}^{m} h_{n, m, a}\left(\begin{array}{c}
n+t-a \\
n
\end{array}\right)=\sum_{a=0}^{m} g_{n, m, m-a}\left(\begin{array}{c}
n+t-a \\
n
\end{array}\right) \\
& =\sum_{a=0}^{m}\left(\begin{array}{c}
m \\
m-a
\end{array}\right)\left(\begin{array}{c}
n-m \\
a
\end{array}\right)\left(\begin{array}{c}
t+n-a \\
n
\end{array}\right) \\
& =\sum_{a=0}^{m}\left(\begin{array}{c}
m \\
a
\end{array}\right)\left(\begin{array}{c}
n-m \\
a
\end{array}\right)\left(\begin{array}{c}
t+n-a \\
n
\end{array}\right) \\
& =\left(\begin{array}{c}
t+m \\
m
\end{array}\right)\left(\begin{array}{c}
t+n-m \\
n-m
\end{array}\right) .
\end{aligned}
$$

The last equality is proven in $[\mathbb{R}$, p. 17]; it is an application of the Vandermonde convolution formula. The result follows since $t=[s / 2]$. Note that the summation from 0 to $m$ is correct even when $t$ is smaller than $m$ because $\left(\begin{array}{c}n+t-a \\ n\end{array}\right)=0$ for $a>t$.

Combining the fact that $\chi\left(\mathcal{L}_{m, N_{s}}^{S p}\right)=\left|R_{s m}^{S p}\right|$ with lemma 4.9 and propositions 4.8 and 4.11 we obtain

Corollary 4.12. For any $m \in[0, n]$,

$$
\chi\left(\mathcal{L}_{m, N_{s}}^{S p}\right)=\left(\begin{array}{c}
{[s / 2]+m} \\
m
\end{array}\right)\left(\begin{array}{c}
{[s / 2]+n-m} \\
n-m
\end{array}\right) .
$$

In particular, $\chi\left(\mathcal{L}_{m, N_{s}}^{S p}\right)=\chi\left(\mathcal{L}_{n-m, N_{s}}^{S p}\right)$ and $\chi\left(\mathcal{L}_{0, N_{s}}^{S p}\right)=\chi\left(\mathcal{L}_{n, N_{s}}^{S p}\right)=\left(\begin{array}{c}{[s / 2]+n} \\ n\end{array}\right)$. 
Remarks. 1. The variety $\left(\mathcal{L}_{m, N_{s}}^{S p}\right)$ is isomorphic to the varieties $\left(\mathcal{L}_{m+2 n i, N_{s}}^{S p}\right)$ and $\left(\mathcal{L}_{-m+2 n i, N_{s}}^{S p}\right)$ for any $i \in \mathbf{Z}$ via the maps $L \mapsto \pi^{i} L$ and $L \mapsto \pi^{i} L^{*}$ respectively. Thus, the result is true for all $m \in \mathbf{Z}$ if $m$ is replaced by $m^{\prime} \in[0, n]$ such that $m \equiv \pm m^{\prime}(\bmod 2 n)$.

2. The formula for self-dual lattices can be proven in a much simpler way. Since $r_{n+i}=-r_{i}$ for all $i$, a self-dual lattice $\Lambda_{\mathrm{r}}$ is fixed by $N_{s}$ when $r_{n} \leq r_{n-1} \leq \cdots \leq$ $r_{1} \leq-r_{1} \leq \cdots \leq-r_{n} \leq r_{n}+s$. Setting $d_{i}=-r_{i}$, we have $0=d_{0} \leq d_{1} \leq \cdots \leq$ $d_{n} \leq d_{n+1}=[s / 2]$. This is just an arrangement of [s/2] balls in $n+1$ boxes (the $i$ th box contains $d_{i}-d_{i-1}$ balls), and there are $\left(\begin{array}{c}{[s / 2]+n} \\ n\end{array}\right)$ such arrangements.

We now study the fixed symplectic lattice chains of type $J$ for $J$ a nonempty subset of $[0, n]$. Let $J=\left\{m_{1}<\cdots<m_{l}\right\}$. It is clear from the definition that a lattice chain $\left(L_{m}\right) \in X_{J}^{S p}$ is determined by the $L_{m_{i}}$ 's. Conversely, any sequence $L_{m_{1}} \supset \cdots \supset L_{m_{l}}$ with $L_{m_{i}} \in \mathcal{L}_{m_{i}}^{S p}$ extends uniquely to a chain in $X_{J}^{S p}$. To see this, note that if $L \subset L^{\prime}$, then $L^{*} \supset L^{\prime *}$. Thus, if $L$ and $L^{\prime}$ are both symplectic, the totally ordered set of lattices associated to $L$ and $L^{\prime}$ interlock to form a chain of lattices. This shows that $\left(X_{J, N_{s}}^{S p}\right)^{k^{*}}=\left\{\left(\Lambda_{\mathbf{r}^{m}}\right) \mid\left(\mathbf{r}^{m_{1}}, \ldots, \mathbf{r}^{m_{l}}\right) \in Y_{J s}^{S p}\right\}$ where

$$
Y_{J s}^{S p}=\left\{\left(\mathbf{r}^{m_{1}}, \ldots, \mathbf{r}^{m_{l}}\right) \mid \mathbf{r}^{m_{i}} \in R_{s m_{i}}^{S p} \text { and } \mathbf{r}^{m_{1}} \leq \cdots \leq \mathbf{r}^{m_{l}}\right\}
$$

For $\mathbf{r} \in R_{s m_{1}}^{S p}$, let $Y_{J s}^{S p}(\mathbf{r})$ denote the chains in $Y_{J s}^{S p}$ with $\mathbf{r}^{m_{1}}=\mathbf{r}$.

These sets can be given a uniform combinatorial description in terms of a certain directed graph $\Delta$. The vertex set of $\Delta$ is $C_{s}^{n+1}$, the distinct arrangements of $s$ balls in $n+1$ boxes. We index the boxes from 0 to $n$, and call the number of balls in the $i$ th box $p_{i}$. Arrange the boxes in a line with the index increasing to the right. Viewing a box as two boundary walls, a vertex is just a string of $s$ balls and $n$ walls, both numbered starting from 1 ; the first and last walls are redundant because they do not separate adjacent boxes. Such a string also uniquely determines an arrangement of $n$ walls in $s+1$ cells, where a cell is determined by boundary balls. Let $b_{j}$ be the number of walls in the $j$ th cell for $j \in[0, s]$, with the cell index also increasing to the right.

Two vertices $v$ and $v^{\prime}$ are connected by an edge if one configuration is obtained from the other by moving a ball between two adjacent boxes, i.e. by reversing the order of a wall and ball next to each other. Given a vertex $v$, define $I_{v} \subseteq[1, n]$ recursively by prescribing that for $i \in[0, n-1]$, the size of $\{i, i+1\} \cap I_{v}$ is congruent to $p_{i}$ modulo 2. The recursion can begin because $0 \notin I_{v}$. If $v$ and $v^{\prime}$ are adjacent, then there exists $i \in[0, n-1]$ such that $p_{j}$ and $p_{j}^{\prime}$ have the same parity if and only if $j$ is neither $i$ nor $i+1$. Thus, $I_{v}$ and $I_{v^{\prime}}$ differ only at the single element $i+1$. We assign a direction to the edge from $v$ to $v^{\prime}$ by having it point towards the vertex with the larger associated set, namely, the set containing $i+1$.

The sets $G_{s I}^{S p}$ are disjoint subsets of the vertex set and in fact define a partition of $V(\Delta)$.

Proposition 4.13. The vertex set $V(\Delta)=C_{s}^{n+1}$ is the disjoint union of the $G_{s I}^{S p}$ 's. Furthermore, $\overrightarrow{v v^{\prime}}$ is an edge if and only if there exists $m \in[0, n-1]$ such that $\phi^{-1} \psi^{-1}(v) \in R_{s m}^{S p}, \phi^{-1} \psi^{-1}\left(v^{\prime}\right) \in R_{s, m+1}^{S p}$, and $\phi^{-1} \psi^{-1}(v) \leq \phi^{-1} \psi^{-1}\left(v^{\prime}\right)$.

Proof. Since the $G_{s I}^{S p}$, s are disjoint, in order to prove the first claim, it suffices to show that $v \in G_{s I_{v}}^{S p}$. It is obvious from the construction of $I_{v}$ that $p_{i}$ has the parity 
required of an element of $G_{s I_{v}}^{S p}$ for $i \in[0, n-1]$. It only remains to check that $p_{n}$ is even if and only if $n \in I_{v}$. Since $\sum_{i=0}^{n} p_{n}=s$ is odd, this is a consequence of the following lemma.

Lemma 4.14. Suppose $\sum_{i=0}^{j} p_{i}$ is odd (resp. even). Then $j \notin I_{v}$ if and only if $p_{j}$ is odd (resp. even).

Proof. We use induction on $j$. Since $0 \notin I_{v}$, the statement is vacuous for $j=0$. Assume it is true for $j-1$. Suppose $\sum_{i=0}^{j} p_{i}$ is odd and $p_{j}$ is odd (resp. even). Then $\sum_{i=0}^{j-1} p_{i}$ is even (resp. odd). If $p_{j-1}$ is even, then $j-1 \notin I_{v}$ (resp. $j-1 \in I_{v}$ ) by induction. Since $p_{j-1}$ even also implies that $j-1$ and $j$ are simultaneously in or out of $I_{v}$, we have $j \notin I_{v}$ (resp. $j \in I_{v}$ ). If $p_{j-1}$ is odd, then $j-1 \in I_{v}$ (resp. $j-1 \notin I_{v}$ ), again giving the desired result. The proof is similar for $\sum_{i=0}^{j} p_{i}$ even.

Suppose that $\mathbf{r} \in R_{s m}^{S p}, \mathbf{r}^{\prime} \in R_{s, m+1}^{S p}$, and $\mathbf{r} \leq \mathbf{r}^{\prime}$. Then there exist subsets $I$ and $I^{\prime}$ of $[1, n]$ such that $\mathbf{r} \in R_{s I}^{S p}, \mathbf{r}^{\prime} \in R_{s I^{\prime}}^{S p},|I|=m,\left|I^{\prime}\right|=m+1$, and $I \subset I^{\prime}$. If $i$ is the unique element of $I^{\prime} \backslash I$, then $r_{j}^{\prime}=r_{j}$ except at one of the two indices $i$ and $n+i$ where it is increased by 1 . Let $\mathbf{p}=\psi \phi(\mathbf{r}) \in G_{s I}^{S p}$ and $\mathbf{p}^{\prime}=\psi \phi\left(\mathbf{r}^{\prime}\right) \in G_{s I^{\prime}}^{S p}$. It is immediate that $\mathbf{p}^{\prime}$ is obtained from $\mathbf{p}$ by shifting a ball between the $(i-1)$ th and $i$ th boxes. Since $I \subset I^{\prime}, \overrightarrow{\mathbf{p p}^{\prime}}$ is an edge. The argument is reversible, so the proposition is proved.

Set $m_{0}=0$ and $m_{l+1}=n$. Let $E_{J s}^{n}$ denote the set of sequences of vertices $\left(v_{1}, \ldots, v_{l}\right)$ such that $v_{i} \in G_{s m_{i}}^{S p}$ and there exists a (directed) path in $\Delta$ going through all of the $v_{i}$ 's. In particular, if $J=\left\{m, m+1, \ldots, m^{\prime}\right\}$, then $E_{J s}^{n}$ is the set of paths from $G_{s m}^{S p}$ to $G_{s m^{\prime}}^{S p}$. For each $v \in G_{s m_{i}}^{S p}$, we let $E_{J s}^{n}(v)$ be the subset of $E_{J S}^{n}$ consisting of sequences starting at $v$. Proposition 4.13 implies that there is a bijection $Y_{J s}^{S p} \stackrel{\sim}{\rightarrow} E_{J s}^{n}$ which restricts to bijections $Y_{J s}^{S p}(\mathbf{r}) \sim{ }_{\rightarrow} E_{J s}^{n}(\psi \phi(\mathbf{r}))$. We thus get a concrete combinatorial interpretation of the Euler characteristics of the fixed point varieties with respect to $N_{s}$ on each affine flag manifold:

Proposition 4.15. Suppose that char $k \neq 2$ and $\left(\operatorname{char}^{\prime} k, n\right)=1$. Let $s$ be an odd integer relatively prime to $n$ and $J$ a nonempty subset of $[0, n]$. Then $\chi\left(X_{J, N_{s}}^{S p}\right)=$ $\left|E_{J s}^{n}\right|$.

We define a set

$$
Z_{J s}^{n}=\left\{(v, \sigma) \mid \sigma:[1, n] \rightarrow[0, l], \sigma^{-1}(0)=I_{v} \text {, and }\left|\sigma^{-1}(i)\right|=j_{i} \stackrel{\text { def }}{=} m_{i+1}-m_{i}\right\} .
$$

Let $\theta: E_{J s}^{n} \rightarrow Z_{J s}^{n}$ be the function $\left(v_{1}, v_{2}, \ldots, v_{l}\right) \mapsto\left(v_{1}, \sigma\right)$ where $\sigma^{-1}(0)=I_{v_{1}}$, $\sigma^{-1}(l)=I_{v_{l}}^{c}$, and $\sigma^{-1}(i)=I_{v_{i+1}}-I_{v_{i}}$ for $i \in[1, l-1]$. (Note that $\sigma^{-1}(0)$ and $\sigma^{-1}(l)$ can be empty.) Consequently,

$$
\left|E_{J s}^{n}\right|=\sum_{(v, \sigma) \in Z_{J s}^{n}} \theta^{-1}((v, \sigma)) .
$$

This observation provides an algorithm for computing the Euler characteristics in terms of a game. If $v$ is a vertex viewed as a string of balls and walls, then we obtain all $v^{\prime}$ adjacent to $v$ by replacing some substring $\mid \bullet$ by $\bullet \mid$ or vice versa, where - denotes a ball and $\mid$ a wall. The edge $\overrightarrow{v v^{\prime}}$ is contained in $\Delta$ if and only if the wall involved in the switch is not in $I_{v}$. The elements of $\theta^{-1}((v, \sigma))$ may be found by the following procedure. Find all possible configurations of balls and walls obtained 
from $v$ by one move for each wall in $\sigma^{-1}(1)$. For each such $v_{2}$, repeat the process with the walls in $\sigma^{-1}(2)$. We continue until a ball has been moved over each wall in $\sigma^{-1}([1, l-1])$, thereby getting all $l$-tuples of vertices $\left(v, v_{2}, \ldots, v_{l}\right) \in \theta^{-1}((v, \sigma))$.

In order to evaluate the sum (4-2), we will pass from $Z_{J s}^{n}$ to a more familiar index set consisting of intersection matrices. As a first step, we define a map $\eta$ : $V(\Delta) \rightarrow C_{n}^{t+1}$ where $t=[s / 2]$. Given a vertex $v$, let $\mathbf{b} \in C_{n}^{s+1}$ be the corresponding arrangement of $n$ walls in $s+1$ cells, and set $\eta(v)=\mathbf{a}$ with $a_{k}=b_{2 k}+b_{2 k+1}$. This function simply pools the walls in cells $2 k$ and $2 k+1$ into one larger cell. We let $\eta_{m}$ denote the restriction of $\eta$ to $G_{s m}^{S p}$. We also set $C_{m}^{t+1}(\mathbf{a})=\left\{\left(c_{0}, \ldots, c_{t}\right) \mid c_{k} \in\right.$ $\left[0, a_{k}\right]$ and $\left.\sum_{k=0}^{t} c_{k}=m\right\}$.

Proposition 4.16. There is a bijection between $\eta_{m}^{-1}(\mathbf{a})$ and $C_{m}^{t+1}(\mathbf{a})$; in particular, $\eta_{0}$ is a bijection. Moreover, identifying $G_{s 0}^{S p}$ and $C_{n}^{t+1}$ via $\eta_{0}, \eta(v)$ is the unique element of $G_{s 0}^{S p}$ from which $v$ can be obtained using only moves of the form $|\bullet \rightsquigarrow \bullet|$.

Proof. Suppose that $\mathbf{p} \in G_{s 0}^{S p}$, so that each box contains an even number of balls except for the last one. The $i$ th wall has $p_{0}+\cdots+p_{i-1}$ balls to its left, and since this is an even number, it is in a cell with an even index. Thus, $b_{j}=0$ for $j$ odd. In this case, $a_{k}=b_{2 k}$ and $\eta_{0}$ is injective because $\mathbf{b}$ is uniquely determined by $\mathbf{p}$. Surjectivity follows since $\left|G_{s 0}^{S p}\right|=\left|C_{n}^{t+1}\right|$ by proposition 4.11.

Consider a game starting at $\mathbf{p}$ with only moves of the form $|\bullet \rightsquigarrow \bullet|$ allowed. The only balls which can take part in these switches are the leftmost balls in boxes $1, \ldots, n$. To see this, note that walls can only move to the right. Consequently, the balls in box 0 play no role. Also, if a ball becomes the first ball in its box during the course of the game without starting out in this position, then the wall left of it must already have moved. Since each wall can only move once, the ball is not involved in the game. It follows that no even-numbered balls can move. Thus, $b_{2 k}+b_{2 k+1}$, the number of walls between balls $2 k$ and $2(k+1)$, never changes, i.e. $\eta$ is constant on the set of configurations obtained as outcomes of this game. This proves the uniqueness part of the final statement.

We will show existence of such a path by induction on $m$. This is trivial for $m=0$. Suppose $m \geq 1$ and the claim is true for $m-1$. Take $v=\mathbf{p} \in G_{s m}^{S p}$, and let $i$ be the smallest integer such that $p_{i}$ is odd. Equivalently, $i+1$ is the smallest element of $\hat{I}_{v}$; in fact, $i+1 \in I_{v}$ because $m \neq 0$. Since $p_{i} \neq 0$, the $(i+1)$ st wall is immediately preceded by a ball. We obtain a vertex $v^{\prime}$ adjacent to $v$ by moving this wall to the left, and $\overrightarrow{v^{\prime} v}$ is an edge since $i+1 \in I_{v}$. This implies that, $v^{\prime} \in G_{s, m-1}^{S p}$, so there exists a path to $v^{\prime}$ of the correct form by inductive hypothesis. Concatenating $\overrightarrow{v^{\prime} v}$ with this path proves the result.

Finally, given $\mathbf{c} \in C_{m}^{t+1}(\mathbf{a})$, we obtain a vertex $\tau(\mathbf{c})$ in $G_{s m}^{S p}$ by moving ball $2 k+1$ past the last $c_{k}$ walls in the $2 k$ th cell; such a ball exists because the last cell has odd index. These moves are of the specified type, so $\eta(\tau(\mathbf{c}))=\mathbf{a}$. The map $C_{n m}^{t+1}(\mathbf{a}) \stackrel{\tau}{\rightarrow} \eta_{m}^{-1}(\mathbf{a})$ is surjective by the previous paragraph. It is injective because the $(2 k+1)$ st cell of $\tau(\mathbf{c})$ contains $c_{k}$ walls.

Let $\mathbf{m}_{J}$ be the sequence $\left(0=m_{0}, m_{1}, \ldots, m_{l}, m_{l+1}=n\right)$. We define a map $\xi$ from $Z_{J s}^{n}$ to the set of $\left(\mathbf{m}_{J}, t+1\right)$-intersection matrices $Q_{\mathbf{m}_{J}, t+1}$. (Both row and column indexing start at 0 .) Let $\mathbf{a}=\eta(v)$, and let $A_{k}$ be the set of walls in the 
$2 k$ th cell of $\eta_{0}^{-1}(\mathbf{a})$. Set $\xi(v, \sigma)=\left(\left|\sigma^{-1}(i) \cap A_{k}\right|\right)$ and $\zeta=\xi \circ \theta$. We obtain

$$
\left|E_{J s}^{n}\right|=\sum_{Q \in Q_{\mathbf{m}_{J}, t+1}}\left|\zeta^{-1}(Q)\right| \text {. }
$$

Fix an intersection matrix $Q$. Notice that the only balls which play a role in moves involving the walls in $A_{k}$ are the boundaries of the $2 k$ th cell. Since a ball bounds only one even-numbered cell, this shows that the manipulations of the game take place independently within each $A_{k}$. Consequently, it will suffice to understand the game for the special cases of a string of walls with a boundary ball on both sides or only on the right. These correspond to the game restricted to the $2 k$ th cell for $k>0$ and $k=0$ respectively.

Assume the cell contains $d$ walls and suppose $z_{0}, \ldots, z_{l}$ are nonnegative integers such that $\sum_{i=1}^{l} z_{i}=d$. Consider a game with $z_{i}$ moves at the $i$ th step for $i \in$ $[0, l-1]$, where only moves of the form $|\bullet \rightsquigarrow \bullet|$ are allowed at the 0th step. Call the number of outcomes of the game $\alpha_{\mathbf{z}}^{d}$ if the cell is only bounded on the right and $\beta_{\mathbf{z}}^{d}$ if it is bounded on both sides.

\section{Lemma 4.17.}

$$
\alpha_{\mathbf{z}}^{d}=1 \text { and } \beta_{\mathbf{z}}^{d}=\prod_{i=1}^{l-1}\left(z_{i}+1\right) .
$$

Proof. Suppose there is only a boundary ball on the right. Then there is only one outcome of the game; indeed, we have already seen in the proof of proposition 4.16 that each move consists of the ball shifting one box to the left. Now suppose the cell has a ball on each side. The 0th move is uniquely determined, and setting $z_{0}^{\prime}=0$ and $z_{i}^{\prime}=z_{i}$ for $i \in[1, l]$, we have $\beta_{\mathbf{z}}^{d}=\beta_{\mathbf{z}^{\prime}}^{d-z_{0}}$. We may thus assume that $z_{0}=0$. The left ball can only move to the right and vice versa because otherwise a ball would jump over the same wall twice. Hence, for each $c \in[0, d]$, there are $c+1$ possible configurations after $c$ moves corresponding to $c^{\prime} \in[0, c]$ jumps of the left ball and $c-c^{\prime}$ jumps of the right ball. The resulting strings have the form of the following diagram:

$$
\overbrace{\|\cdots\| \cdot}^{c^{\prime}} \bullet \overbrace{\|\cdots\| \cdot \| \cdot \overbrace{\|\cdots\|}^{d-c}}^{c-c^{\prime}} .
$$

The outer walls have all been used, so the $c$ moves reduce the game to the game of the same form with $d-c$ walls. The lemma follows from these observations by induction.

\section{Corollary 4.18.}

$$
\left|\zeta^{-1}(Q)\right|=\prod_{i=1}^{l-1} \prod_{k=1}^{t}\left(q_{i k}+1\right)
$$

Proof. Let $\mathbf{q}^{k}$ be the $k$ th column of $Q$. Then $\left|\zeta^{-1}(Q)\right|=\alpha_{\mathbf{q}^{0}}^{a_{0}} \prod_{k=1}^{t} \beta_{\mathbf{q}^{k}}^{a_{k}}$, and lemma 4.17 completes the proof.

Let $\gamma_{d}^{t}=\sum_{\mathbf{y} \in C_{d}^{t}} \prod_{k=1}^{t}\left(y_{k}+1\right)$. We need the following lemma:

Lemma 4.19. For any $d \geq 0$ and $t>0$,

$$
\gamma_{d}^{t}=\left(\begin{array}{c}
2 t+d-1 \\
d
\end{array}\right)
$$


Proof. We use induction on $t$. The claim is true for $t=1$, since $\gamma_{d}^{1}=d+1=\left(\begin{array}{c}d+1 \\ d\end{array}\right)$. Now take $t>1$ and assume that the result holds for $t-1$. Then

$$
\begin{aligned}
\gamma_{d}^{t}=\sum_{\mathbf{y} \in C_{d}^{t}} \prod_{k=1}^{t}\left(y_{k}+1\right) & =\sum_{y_{d}=0}^{d}\left(y_{d}+1\right) \sum_{\mathbf{x} \in C_{d-y_{d}}^{t-1}} \prod_{k=1}^{t-1}\left(x_{k}+1\right) \\
& =\sum_{y_{d}=0}^{d}\left(y_{d}+1\right)\left(\begin{array}{c}
2(t-1)+d-y_{d}-1 \\
d-y_{d}
\end{array}\right) \\
& =\sum_{y_{d}=0}^{d}\left(\begin{array}{c}
y_{d}+1 \\
y_{d}
\end{array}\right)\left(\begin{array}{c}
2(t-1)+d-y_{d}-1 \\
d-y_{d}
\end{array}\right) \\
& =\left(\begin{array}{c}
2 t+d-1 \\
d
\end{array}\right)
\end{aligned}
$$

where the last step is a version of the Vandermonde convolution formula $\underline{R}$ p. $10]$.

Corollary 4.20. For any $d \geq 0$ and $t>0$,

$$
\sum_{\mathbf{z} \in C_{d}^{t+1}} \prod_{k=1}^{t}\left(z_{k}+1\right)=\left(\begin{array}{c}
2 t+d \\
d
\end{array}\right)
$$

Proof. We have

$$
\sum_{\mathbf{z} \in C_{d}^{t+1}} \prod_{k=1}^{t}\left(z_{k}+1\right)=\sum_{z_{d}=0}^{d} \gamma_{d-z_{d}}^{t}=\sum_{z_{d}=0}^{d}\left(\begin{array}{c}
2 t+d-z_{d}-1 \\
d-z_{d}
\end{array}\right)=\left(\begin{array}{c}
2 t+d \\
d
\end{array}\right)
$$

the last equality is a standard binomial identity $[\mathrm{R}$, p. 7$]$.

We can now calculate $\left|E_{J s}^{n}\right|$.

\section{Proposition 4.21.}

$$
\left|E_{J s}^{n}\right|=\left(\begin{array}{c}
t+j_{0} \\
t
\end{array}\right)\left(\begin{array}{c}
t+j_{l} \\
t
\end{array}\right) \prod_{i=1}^{l-1}\left(\begin{array}{c}
s+j_{i}-1 \\
j_{i}
\end{array}\right) .
$$

Proof. Let $\mathbf{q}_{i}$ be the $i$ th row of $Q$. Recall from lemma 3.9 that the map $Q \mapsto$ $\left(\mathbf{q}_{0}, \ldots, \mathbf{q}_{l}\right)$ is a bijection of $Q_{\mathbf{m}_{J}, s}$ onto $\prod_{i=0}^{l} C_{j_{i}}^{t+1}$. From (4-3) and corollaries 4.18 and 4.20 we have

$$
\begin{aligned}
\left|E_{J s}^{n}\right| & =\sum_{Q \in Q_{\mathbf{m}_{J}, t+1}}\left|\zeta^{-1}(Q)\right|=\sum_{\substack{\left(\mathbf{q}_{0}, \ldots, \mathbf{q}_{l}\right) \in \in \\
\prod_{i=0}^{l} C_{j_{i}}^{t+1}}} \prod_{i=1}^{l-1} \prod_{k=1}^{t}\left(q_{i k}+1\right) \\
& =\left|C_{j_{0}}^{t+1}\right|\left|C_{j_{l}}^{t+1}\right| \prod_{i=1}^{l-1} \sum_{\mathbf{q}_{i} \in C_{j_{i}}^{t+1}} \prod_{k=1}^{t}\left(q_{i k}+1\right)=\left(\begin{array}{c}
t+j_{0} \\
t
\end{array}\right)\left(\begin{array}{c}
t+j_{l} \\
t
\end{array}\right) \prod_{i=1}^{l-1} \gamma_{j_{i}}^{t+1} \\
& =\left(\begin{array}{c}
t+j_{0} \\
t
\end{array}\right)\left(\begin{array}{c}
t+j_{l} \\
t
\end{array}\right) \prod_{i=1}^{l-1}\left(\begin{array}{c}
2 t+j_{i} \\
j_{i}
\end{array}\right)=\left(\begin{array}{c}
t+j_{0} \\
t
\end{array}\right)\left(\begin{array}{c}
t+j_{l} \\
t
\end{array}\right) \prod_{i=1}^{l-1}\left(\begin{array}{c}
s+j_{i}-1 \\
j_{i}
\end{array}\right) .
\end{aligned}
$$

Combining this result with proposition 4.15 gives the following theorem: 
Theorem 4.22. Suppose that char $k \neq 2$ and $\left(\operatorname{char}^{\prime} k, n\right)=1$. Let $s$ be an odd integer relatively prime to $n$ with $t=[s / 2]$ and $J=\left\{m_{1}<\cdots<m_{l}\right\}$ a nonempty subset of $[0, n]$. Let $j_{i}=m_{i+1}-m_{i}$ where $m_{0}=0$ and $m_{l+1}=n$. Then

$$
\chi\left(X_{J, N_{s}}^{S p}\right)=\left(\begin{array}{c}
t+j_{0} \\
t
\end{array}\right)\left(\begin{array}{c}
t+j_{l} \\
t
\end{array}\right) \prod_{i=1}^{l-1}\left(\begin{array}{c}
s+j_{i}-1 \\
j_{i}
\end{array}\right)
$$

In particular, $\chi\left(\hat{\mathcal{B}}_{N_{s}}\right)=s^{n}$ and $\chi\left(\mathcal{L}_{m, N_{s}}^{S p}\right)=\left(\begin{array}{c}t+j_{0} \\ t\end{array}\right)\left(\begin{array}{c}t+j_{l} \\ t\end{array}\right)$.

Proof. It only remains to check the final statement. The symplectic lattices of type $m$ correspond to $J=\{m\}$, so $l=1, j_{0}=m$, and $j_{1}=n-m$. Substituting, we obtain another proof of corollary 4.12 The full affine flag manifold is isomorphic to $X_{[0, n]}^{S p}$. In this case, $l=n+1, j_{0}=j_{n+1}=0$, and $j_{i}=1$ for $i \in[1, n]$, and the result follows.

Remark. As in the case of the root system $A_{n}$, the Euler characteristic is just the number of simplices of the type corresponding to the parahoric class within the subset of the positive Weyl chamber given by $\lambda_{0} \leq s$, where $\lambda_{0}$ is the affine root.

Corollary 4.23. Under the hypotheses of the previous theorem,

$$
\chi\left(X_{J, N_{s}}^{S p}\right) \leq \chi\left(X_{J \cup(2 n-J) \backslash\{2 n\}, N_{s}}\right),
$$

with equality for $n=1$ or $s=1$ and strict inequality otherwise.

Proof. The inequality follows immediately from the fact that $\left(X_{J, N_{s}}^{S p}\right)^{k^{*}}$ is a subset of the finite set $\left(X_{J \cup(2 n-J) \backslash\{2 n\}, N_{s}}\right)^{k^{*}}$. These sets are equal when $n=1$ because $S p_{2}(k)=S L_{2}(k)$; thus the formulas for the Euler characteristics of the affine flag varieties of $S p_{2}(F)$ given in theorem 4.22 agree with those given in theorem 3.13 Also, if $s=1$, then the Euler characteristics are 1 for both the symplectic and special linear groups.

Now suppose that $n$ and $s$ are both greater than 1 . We have

$$
\chi\left(X_{J \cup(2 n-J) \backslash\{2 n\}, N_{s}}\right)=s^{-1}\left(\begin{array}{c}
2 t+2 j_{0} \\
2 t
\end{array}\right)\left(\begin{array}{c}
2 t+2 j_{l} \\
2 t
\end{array}\right) \prod_{i=1}^{l-1}\left(\begin{array}{c}
s+j_{i}-1 \\
j_{i}
\end{array}\right)^{2} .
$$

Note that $\left(\begin{array}{c}2 t+2 q \\ 2 t\end{array}\right)=\left(\begin{array}{c}t+q \\ t\end{array}\right)$ for $q=0$ and

$$
\left(\begin{array}{c}
2 t+2 q \\
2 t
\end{array}\right)=\left(\begin{array}{c}
t+q \\
t
\end{array}\right) \prod_{p=1}^{q} \frac{2 t+2 p-1}{2 p-1} \geq s\left(\begin{array}{c}
t+q \\
t
\end{array}\right)
$$

for $q \geq 1$. Since $t \geq 1$, the inequality is strict when $q \geq 2$. Furthermore, $\left(\begin{array}{c}s+q-1 \\ q\end{array}\right)^{2} \geq$ $s\left(\begin{array}{c}s+q-1 \\ q\end{array}\right)$ for $q \geq 1$, with strict equality for $q \geq 2$. This proves the claim if $j_{i} \geq 2$ for any $i \in[0, l]$. Otherwise, $\sum_{i=0}^{l} j_{i}=n \geq 2$ implies that there exist two indices $i_{1}$ and $i_{2}$ such that $j_{i_{1}}=j_{i_{2}}=1$. The result follows because $s^{2}>s$.

We can also obtain a combinatorial description of the Euler characteristics of the fixed point varieties in the classical symplectic flag manifolds for certain nilpotent conjugacy classes of $\mathfrak{s p}_{2 n}(k)$. The nilpotent conjugacy classes of $\mathfrak{s p}_{2 n}(k)$ are characterized by symplectic partitions of $2 n$, i.e. partitions whose odd parts come in pairs $\mathrm{Sp}$. These partitions are the dimensions of the Jordan blocks of the nilpotent element of $\mathfrak{s p}_{2 n}(k)$. Let $\mathcal{C}$ be a nilpotent conjugacy class corresponding to a partition 
with at most one part appearing with odd multiplicity. Such a class is determined by $n_{0} \in[0, n]$ and a partition $n_{1}, \ldots, n_{l}$ of $n-n_{0}$. Let $M_{0}$ and $M_{i}$ be matrices with a single Jordan block in $\mathfrak{s p}_{2 n_{0}}(k)$ and $\mathfrak{g l}_{n_{i}}(k)$ respectively. Recall that $\mathfrak{g l}_{n}(k)$ injects into $\mathfrak{s p}_{2 n}(k)$ via $M \mapsto \operatorname{diag}\left(M,-M^{t}\right)$. Then the image of $\left(M_{0}, M_{1}, \ldots, M_{l}\right)$ under the injections $\mathfrak{s p}_{2 n_{0}}(k) \times \prod_{i=1}^{t} \mathfrak{g l}_{n_{i}}(k) \hookrightarrow \prod_{i=0}^{l} \mathfrak{s p}_{2 n_{i}}(k) \hookrightarrow \mathfrak{s p}_{2 n}(k)$ is an element in this class; it has two Jordan blocks of size $n_{i}$ and one of size $2 n_{0}$.

Theorem 4.24. Assume that char $k \neq 2$ and $\left(n, \operatorname{char}^{\prime} k\right)=1$. Let $N \in \mathfrak{s p}_{2 n}(k)$ be an element of a nilpotent conjugacy class with at most one Jordan block size having odd multiplicity, and let $2 n=2 n_{0}+2 \sum_{i=1}^{l} n_{i}$ be the corresponding symplectic partition of $2 n$. Let $s=2 t+1$ be any odd integer greater than $2 l$ and relatively prime to $n$. Define $\mathbf{a} \in C_{n}^{t+1}$ by $a_{k}=n_{k}$ for $k \in[0, l]$ and $a_{k}=0$ for $k>l$. Then for each nonempty $J \subseteq[1, n]$,

$$
\chi\left(\mathcal{F}_{J, N}^{S p}\right)=\left|E_{J \cup\{0\}, s}^{n}\left(\eta^{-1}(\mathbf{a})\right)\right|=\sum_{Q \in Q_{\mathbf{m}_{J}, t+1}(\mathbf{a})} \prod_{i=1}^{l-1} \prod_{k=1}^{t}\left(q_{i k}+1\right) .
$$

In particular,

$$
\chi\left(\mathcal{B}_{N}^{S p}\right)=\frac{n ! 2^{n_{1}+\cdots+n_{l}}}{n_{0} ! \cdots n_{l} !} .
$$

Proof. Let $\mathbf{r} \in R_{s 0}^{S p}$ correspond to $\mathbf{a}$, so that setting $\mathbf{q}=\phi(\mathbf{r})$ and $\mathbf{p}=\psi(\mathbf{q})$, we have $\eta_{0}(\mathbf{p})=\mathbf{a}$. We show that the nilpotent map $\bar{N}_{s}$ induced on $W=\Lambda_{\mathbf{r}} / \pi \Lambda_{\mathbf{r}}$ has Jordan block structure given by $n_{0}, \ldots, n_{l}$. It is clear from the definition that $\eta_{0}^{-1}(\mathbf{a})$ is the string of $s+1$ balls and $n$ walls with $n_{k}$ walls in the $2 k$ th cell for $k \in[0, l]$ and the other cells empty. Thus, the nonempty boxes are precisely those numbered $\sum_{i=0}^{d} n_{i}$ for $d \in[0, l]$. The nonzero $q_{i}$ 's then have indices $n$ and $\pm \sum_{i=0}^{d} n_{i}$ for $d \in[0, l-1]$. As shown in the proof of theorem 3.11, a Jordan block of size $d$ corresponds to $j \in[-(n-1), n]$ such that $q_{j}=q_{j+d}=0$ and $q_{j+d^{\prime}} \geq 1$ for $0<d^{\prime}<d$. (It is not necessary to understand the addition of the indices modulo $2 n$ because $q_{n} \neq 0$.) It is immediate that there is one block of size $2 n_{0}$ and two of size $n_{i}$ for each $i \in[1, l]$ and that the block structure comes from an injection of the form $\mathfrak{s p}_{2 n_{0}}(k) \times \prod_{i=1}^{t} \mathfrak{g l}_{n_{i}}(k) \hookrightarrow \mathfrak{s p}_{2 n}(k)$ as described above. The first statement follows, since

$$
\begin{aligned}
\chi\left(\mathcal{F}_{J, N}^{S p}\right)=\left|E_{J \cup\{0\}, s}^{n}\left(\eta^{-1}(\mathbf{a})\right)\right| & =\sum_{Q \in Q_{\mathbf{m}_{J}, t+1}(\mathbf{a})}\left|\zeta^{-1}(Q)\right| \\
& =\sum_{Q \in Q_{\mathbf{m}_{J}, t+1}(\mathbf{a})} \prod_{i=1}^{l-1} \prod_{k=1}^{t}\left(q_{i k}+1\right) .
\end{aligned}
$$

Now suppose $J=[1, n]$. A $\left(\mathbf{m}_{[0, n]}, s\right)$-intersection matrix has a single 1 in each row, so there are $\frac{n !}{a_{0} ! \cdots a_{t} !}$ elements $Q \in Q_{\mathbf{m}_{[0, n]}, s}(\mathbf{a})$. Also, $\left|\zeta^{-1}(Q)\right|=2^{a_{1}+\cdots+a_{t}}$, and since this is constant on the fiber over $\mathbf{a}, \chi\left(\mathcal{B}_{N}^{S p}\right)=\frac{n ! 2^{a_{1}+\cdots+a_{t}}}{a_{0} ! \cdots a_{t} !}=\frac{n ! 2^{n_{1}+\cdots+n_{l}}}{n_{0} ! \cdots n_{l} !}$.

Remarks. 1. Since $\frac{n ! 2^{a_{1}+\ldots a_{t}}}{a_{0} ! \cdots a_{t} !}$ is the term corresponding to $\mathbf{a}$ in the multinomial expansion of $s^{n}=(1+2+\cdots+2)^{n}$, we obtain another proof of the formula for the Euler characteristic of the full affine flag manifold. 
2. The Euler characteristic of each $\mathcal{P}_{N}$ can be obtained from the game with $2 n+1$ balls.

Corollary 4.25. Assume char $k \neq 2$ and $\left(n\right.$, $\left.\operatorname{char}^{\prime} k\right)=1$. Let $N \in \mathfrak{s p}_{2 n}(k)$ be an element of a nilpotent conjugacy class with at most one Jordan block size having odd multiplicity. Then for each nonempty $J \subseteq[1, n], \chi\left(\mathcal{F}_{J, N}^{S p}\right) \leq \chi\left(\mathcal{F}_{J \cup(2 n-J)}\right)$.

Proof. The proof is similar to the first part of corollary 4.23 ,

Remark. The formulas given in theorems 4.24 and 3.11 coincide for $\operatorname{dim} V=2$.

\section{REFERENCES}

[B-B1] A. Bialynicki-Birula, On fixed point schemes of actions of multiplicative and additive groups, Topology 12 (1973), 99-103. MR 47:1816

[B-B2] A. Bialynicki-Birula, Some theorems on actions of algebraic groups, Annals of Math. 98 (1973), 480-497. MR 51:3186

[B-B3] A. Bialynicki-Birula, Some properties of the decompositions of algebraic varieties determined by actions of a torus, Bulletin de L'Académie Polonaise des sciences 24 (1976), 667-674. MR 56:12020

[Bo] A. Borel, Linear algebraic groups, Graduate Texts in Math. 126, Springer-Verlag, Berlin, 1991. MR 92d:20001

[B1] N. Bourbaki, Algèbre, Chap. IX, Hermann, Paris, 1959.

[B2] N. Bourbaki, Algebra II, Chapters 4-7, Springer-Verlag, Berlin, 1990. MR 91h:00003

[KL] D. Kazhdan and G. Lusztig, Fixed point varieties on affine flag manifolds, Israel J. Math. 62 (1988), 129-168. MR 89m:14025

[LS] G. Lusztig and J. M. Smelt, Fixed point varieties on the space of lattices, Bull. London Math. Soc. 23 (1991), 213-218. MR 93e:14065

[R] J. Riordan, Combinatorial identities, Robert E. Krieger Publishing Company, Huntington, New York, 1979. MR 80k:05001

[S] D. S. Sage, The geometry of fixed point varieties on affine flag manifolds, Ph.D. thesis, University of Chicago, 1995.

[S1] J. -P. Serre, Corps locaux, Hermann, Paris, 1962. MR 27:133

[S2] J. -P. Serre, Cohomologie galoisienne, Lecture Notes in Math. 5, Springer-Verlag, Berlin, fourth ed., 1973.

[Sp] N. Spaltenstein, Polynomials over local fields, nilpotent orbits and conjugacy classes in Weyl groups, Astérisque 168 (1988), 191-217. MR 90k:20069

[SS] T. A. Springer and R. Steinberg, Conjugacy classes, Seminar on Algebraic Groups and Related Finite Groups, Lecture Notes in Math. 131, Springer-Verlag, Berlin, 1970, pp. 167-266. MR 42:3091

[St] R. Steinberg, Regular elements of semisimple algebraic groups, Inst. Hautes Études Sci. Publ. Math. 25 (1965), 49-80. MR 31:4788

Department of Mathematics, University of Utah, Salt Lake City, Utah 84112

Current address: School of Mathematics, Institute for Advanced Study, Princeton, New Jersey 08540

E-mail address: sage@ias.edu 